\title{
First lattice calculation of radiative leptonic decay rates of pseudoscalar mesons
}

\author{
A. Desiderio $\odot,{ }^{1}$ R. Frezzotti $\odot,{ }^{1}$ M. Garofalo $\odot,{ }^{2}$ D. Giusti $\odot,{ }^{3,4}$ M. Hansen $\odot,{ }^{5}$ V. Lubicz $\odot,{ }^{2}$ \\ G. Martinelliø, ${ }^{6}$ C. T. Sachrajda, ${ }^{7}$ F. Sanfilippo, ${ }^{4}$ S. Simula $\odot,{ }^{4}$ and N. Tantalo ${ }^{1}$ \\ ${ }^{1}$ Dipartimento di Fisica and INFN, Università di Roma "Tor Vergata," Via della Ricerca Scientifica 1, \\ I-00133 Roma, Italy \\ ${ }^{2}$ Dipartimento di Fisica, Università Roma Tre and INFN, Sezione di Roma Tre, Via della Vasca Navale 84, \\ I-00146 Rome, Italy \\ ${ }^{3}$ Universität Regensburg, Fakultät für Physik, Universitätsstrasse 31, 93040 Regensburg, Germany \\ ${ }^{4}$ Istituto Nazionale di Fisica Nucleare, Sezione di Roma Tre, Via della Vasca Navale 84, \\ I-00146 Rome, Italy \\ ${ }^{5}$ SDU eScience Center, University of Southern Denmark, Campusvej 55, DK-5230 Odense M, Denmark \\ ${ }^{6}$ Physics Department and INFN Sezione di Roma La Sapienza Piazzale Aldo Moro 5, 00185 Roma, Italy \\ ${ }^{7}$ Department of Physics and Astronomy, University of Southampton, \\ Southampton SO17 1BJ, United Kingdom
}

(Received 26 June 2020; accepted 4 November 2020; published 4 January 2021)

\begin{abstract}
We present a nonperturbative lattice calculation of the form factors which contribute to the amplitudes for the radiative decays $P \rightarrow \ell \bar{\nu}_{\ell} \gamma$, where $P$ is a pseudoscalar meson and $\ell$ is a charged lepton. Together with the nonperturbative determination of the corrections to the processes $P \rightarrow \ell \bar{\nu}_{\ell}$ due to the exchange of a virtual photon, this allows accurate predictions at $O\left(\alpha_{\mathrm{em}}\right)$ to be made for leptonic decay rates for pseudoscalar mesons ranging from the pion to the $D_{s}$ meson. We are able to separate unambiguously and nonpertubatively the pointlike contribution, from the structure-dependent, infrared-safe, terms in the amplitude. The fully nonperturbative $O(a)$ improved calculation of the inclusive leptonic decay rates will lead to the determination of the corresponding Cabibbo-Kobayashi-Maskawa matrix elements also at $O\left(\alpha_{\mathrm{em}}\right)$. Prospects for a precise evaluation of leptonic decay rates with emission of a hard photon are also very interesting, especially for the decays of heavy $D$ and $B$ mesons for which currently only modeldependent predictions are available to compare with existing experimental data.
\end{abstract}

DOI: $10.1103 /$ PhysRevD.103.014502

\section{INTRODUCTION}

The unitarity of the Cabibbo-Kobayashi-Maskawa (CKM) matrix is one of the most precise tests of the Standard Model. Indeed, CKM unitarity may rule out many theoretically well-motivated models for new physics and put severe constraints on the energy scale where new phenomena might occur, well beyond the range accessible to direct experimental searches. In this respect, leptonic decay rates of light and heavy pseudoscalar mesons are essential ingredients for the extraction of the CKM matrix elements. A first-principles calculation of these quantities requires nonperturbative accuracy and hence numerical lattice simulations. Moreover, in order to fully exploit the presently available experimental information and to

Published by the American Physical Society under the terms of the Creative Commons Attribution 4.0 International license. Further distribution of this work must maintain attribution to the author(s) and the published article's title, journal citation, and DOI. Funded by SCOAP. perform the next generation of flavor-physics tests, $O\left(\alpha_{\mathrm{em}}\right)$ electromagnetic corrections must be included. In this endeavor, the radiative leptonic decays $P \rightarrow \ell \bar{\nu}_{\ell}(\gamma)$ (where $P$ is a negatively charged pseudoscalar meson, $\ell$ a lepton, $\bar{\nu}_{\ell}$ the corresponding antineutrino, and $\gamma$ a photon) are particularly important; see [1].

Knowledge of the radiative leptonic decay rate in the region of small (soft) photon energies is required in order to properly define the infrared-safe measurable decay rate for the process $P \rightarrow \ell \overline{\boldsymbol{\nu}}_{\ell}(\gamma)$. Indeed, according to the wellknown Bloch-Nordsieck mechanism [2], the integral of the radiative decay rate in the phase space region corresponding to soft photons must be added to the decay rate with no real photons in the final states (the so-called virtual rate) in order to cancel infrared divergent contributions appearing in unphysical quantities at intermediate stages of the calculations.

On the one hand, in the limit of ultrasoft photon energy, the radiative decay rate can be reliably calculated in an effective theory in which the meson is treated as a pointlike particle. This is a manifestation of the well-known 
mechanism known as the "universality of infrared divergences" (see e.g. Refs. [3,4]) that finds its physical explanation in the fact that ultrasoft photons cannot resolve the internal structure of the meson. On the other hand, the ultrasoft limit is an idealization and experimental measurements, particularly in the case of heavy mesons, are inclusive up to photon energies that may be too large to safely neglect the structure-dependent (SD) corrections to the pointlike approximation.

In the region of hard (experimentally detectable) photon energies, radiative leptonic decays represent important probes of the internal structure of the mesons. Moreover, radiative decays can provide independent determinations of CKM matrix elements with respect to the purely leptonic channels. A nonperturbative calculation of the radiative decay rates can be particularly important for heavy mesons since, unlike the case of pions and kaons where such decays have been studied using chiral perturbation theory (ChPT) [5-9], no model-independent calculations have ever been performed. Even in the case of light mesons, although the quoted ChPT calculations represent a firstprinciples approach to the problem, the low-energy constants entering in the final results at $O\left(p^{6}\right)$ have been estimated in phenomenological analyses relying in part on model-dependent assumptions.

In Ref. [10], a strategy to compute QED radiative corrections to the $P \rightarrow \ell \bar{\nu}_{\ell}(\gamma)$ decay rates at $O\left(\alpha_{\mathrm{em}}\right)$ by starting from first-principles lattice calculations was proposed. The strategy has subsequently been applied in Refs. [11-15], within the RM123 approach [16,17], to provide the first nonperturbative model-independent calculation of the decay rates $\pi^{-} \rightarrow \mu^{-} \bar{\nu}_{\mu}(\gamma)$ and $K^{-} \rightarrow \mu^{-} \bar{\nu}_{\mu}(\gamma)$. In these calculations, the real soft-photon contributions have been evaluated in the pointlike effective theory and, using the ChPT results quoted above, the SD corrections have been estimated to be negligible for these processes (see [10]). In the same phenomenological analysis, it has been shown that the SD corrections might instead be relevant for the decays of pions and kaons into electrons. Moreover, by using the same single-pole dominance approximation as originally used in Ref. [18], SD contributions have been estimated to be phenomenologically important for decays of heavy-flavor mesons.

In this paper, we present the first nonperturbative lattice calculation of the rates for the radiative decays $P \rightarrow \ell \bar{\nu} \gamma$,

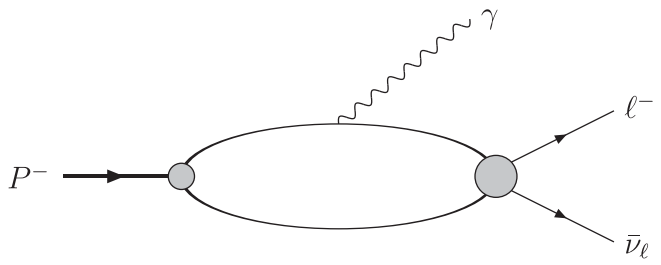

where $P$ is a pion, kaon, $D$ or $D_{s}$ meson. We use the $N_{f}=$ $2+1+1$ gauge ensembles generated by the European Twisted Mass Collaboration (ETMC) and analyzed for mesonic observables in Ref. [19]. Preliminary results from this study were presented in Ref. [20]; the decays of bottom mesons will be studied in future papers. Note also that Kane et al. have presented preliminary results for the decays $D_{s}^{+} \rightarrow \ell^{+} \nu \gamma$ and $K^{-} \rightarrow \ell^{-} \bar{\nu} \gamma$, where $\ell^{ \pm}$represents the charged leptons and $\gamma$ is a hard photon with energy in the range of about $0.5-1 \mathrm{GeV}$ in Ref. [21].

The plan of the remainder of this paper is as follows. In Sec. II, we introduce the basic quantities which enter in the amplitude for the leptonic decay of a pseudoscalar meson with the emission of a real photon; in particular, we define the axial and vector form factors $F_{A}$ and $F_{V}$. We express the decay rates in terms of these quantities in Appendix A. In Sec. III, we describe the general strategy that we followed to extract the amplitudes from suitable Euclidean correlation functions and discuss finite-time effects. The presence of discretization effects which diverge at small photon momenta is demonstrated in Sec. IV and Appendix C, together with a strategy for subtracting them nonperturbatively. In Sec. V, we present the numerical results for pions, kaons, $D$ and $D_{s}$ mesons. Many formulas which are used in the paper are discussed and derived in Appendices A-C. Finally, in Appendix D, we present some of our numerical results, including the correlation matrices, in a way which we hope may be useful to readers who wish to use them in phenomenological applications.

\section{DEFINITION OF THE FORM FACTORS}

The nonperturbative contribution to the radiative leptonic decay rate for the processes $P \rightarrow \ell \bar{\nu}_{\ell} \gamma$ is encoded in the following hadronic matrix element, see the left panel of Fig. 1:

$$
\begin{aligned}
H_{W}^{\alpha r}(k, \boldsymbol{p}) & =\epsilon_{\mu}^{r}(k) H_{W}^{\alpha \mu}(k, \boldsymbol{p}) \\
& =\epsilon_{\mu}^{r}(k) \int d^{4} y e^{i k \cdot y} \mathrm{~T}\left\langle 0\left|j_{W}^{\alpha}(0) j_{\mathrm{em}}^{\mu}(y)\right| P(\boldsymbol{p})\right\rangle,
\end{aligned}
$$

where $\epsilon_{\mu}^{r}(k)$ is the polarization vector of the outgoing photon with four-momentum $k, \boldsymbol{p}$ is the momentum of the ingoing pseudoscalar meson of mass $m_{P}(p \equiv(E, \boldsymbol{p})$, $E=\sqrt{m_{P}^{2}+\boldsymbol{p}^{2}}$, and $\left.p^{2}=m_{P}^{2}\right)$. Here and in the following

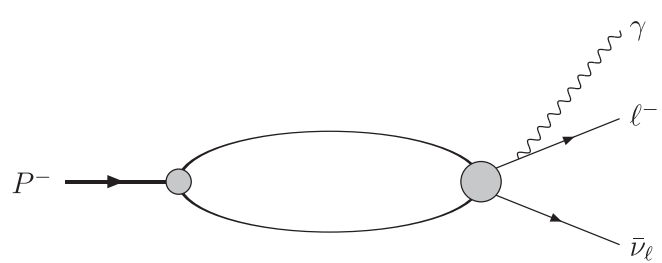

FIG. 1. Feynman diagrams representing the amplitudes with the emission of a real photon from the $P^{-}$meson (left panel) or from the final-state charged lepton $\ell^{-}$(right panel). 
Greek letters, $\alpha, \mu, \nu, \ldots$, denote Lorentz indices, Latin letters, $i, j, k, \ldots$ denote spatial indices, and $r$ denotes the index identifying the polarization of the real photon. The operators

$$
\begin{aligned}
j_{\mathrm{em}}^{\mu}(x) & =\sum_{f} q_{f} \bar{\psi}_{f}(x) \gamma^{\mu} \psi_{f}(x), \\
j_{W}^{\alpha}(x) & =j_{V}^{\alpha}(x)-j_{A}^{\alpha}(x)=\bar{\psi}_{U}(x)\left(\gamma^{\alpha}-\gamma^{\alpha} \gamma_{5}\right) \psi_{D}(x)
\end{aligned}
$$

are, respectively, the electromagnetic hadronic current and the hadronic weak current expressed in terms of the quark fields $\psi_{f}$ having electric charge $q_{f}$ in units of the charge of the positron; $\psi_{U}$ and $\psi_{D}$ indicate the fields of an up-type or a down-type quark and for the mesons considered in this study $U$ can be either an up or a charm quark and $D$ a down or a strange quark. In order to calculate the full amplitude, one has also to consider the contribution in which the photon is emitted from the final-state charged lepton; see the right panel of Fig. 1. This latter contribution, however, can be computed in perturbation theory using the meson's decay constant $f_{P}$. Both contributions are included in the formulas for the decay rate given in Appendix A.

The decomposition of $H_{W}^{\alpha r}(k, \boldsymbol{p})$ in terms of scalar form factors has been discussed in Ref. [10] (see also [22]). Here we adopt the same basis used in that paper to write

$$
\begin{aligned}
H_{W}^{\alpha r}(k, \boldsymbol{p})= & \epsilon_{\mu}^{r}(k)\left\{H_{1}\left[k^{2} g^{\mu \alpha}-k^{\mu} k^{\alpha}\right]\right. \\
& +H_{2}\left[\left(p \cdot k-k^{2}\right) k^{\mu}-k^{2}(p-k)^{\mu}\right](p-k)^{\alpha} \\
& -i \frac{F_{V}}{m_{P}} \varepsilon^{\mu \alpha \gamma \beta} k_{\gamma} p_{\beta} \\
& +\frac{F_{A}}{m_{P}}\left[\left(p \cdot k-k^{2}\right) g^{\mu \alpha}-(p-k)^{\mu} k^{\alpha}\right] \\
& \left.+f_{P}\left[g^{\mu \alpha}+\frac{(2 p-k)^{\mu}(p-k)^{\alpha}}{2 p \cdot k-k^{2}}\right]\right\} .
\end{aligned}
$$

The term in the last line of Eq. (3), which we write as $H_{\mathrm{pt}}^{\alpha \mu}(k, \boldsymbol{p})$, is the pointlike infrared-divergent contribution. The other terms correspond to the so-called SD contribution, $H_{\mathrm{SD}}^{\alpha \mu}(k, \boldsymbol{p}) . H_{\mathrm{pt}}^{\alpha \mu}(k, \boldsymbol{p})$ saturates the Ward Identity (WI) satisfied by $H_{W}^{\alpha \mu}(k, \boldsymbol{p})$,

$k_{\mu} H_{W}^{\alpha \mu}(k, \boldsymbol{p})=k_{\mu} H_{\mathrm{pt}}^{\alpha \mu}(k, \boldsymbol{p})=i\left\langle 0\left|j_{W}^{\alpha}(0)\right| P(\boldsymbol{p})\right\rangle=f_{P} p^{\alpha}$,

$k_{\mu} H_{\mathrm{SD}}^{\alpha \mu}(k, \boldsymbol{p})=0$,

as explained in detail in Appendix C. The four form factors $H_{1,2}$ and $F_{V, A}$ are scalar functions of Lorentz invariants, $m_{P}^{2}, p \cdot k$, and $k^{2}$. Equation (3) is valid for generic (offshell) values of the photon momentum and for generic choices of the polarization vectors. The knowledge of the four form factors in the case of off-shell photons $\left(k^{2} \neq 0\right)$ gives access to the study of decays in which the pseudoscalar meson decays into four leptons. These processes are very interesting in the search of physics beyond the Standard Model and will be the subject of a future work. In this paper, we concentrate on the case in which the photon is on-shell.

By setting $k^{2}=0$, at fixed meson mass, the form factors are functions of $p \cdot k$ only. Moreover, by choosing a physical basis for the polarization vectors so that

$$
\epsilon_{r}(\boldsymbol{k}) \cdot k=0,
$$

one has

$$
\begin{aligned}
H_{W}^{\alpha r}(k, \boldsymbol{p})= & \epsilon_{\mu}^{r}(\boldsymbol{k})\left\{-i \frac{F_{V}}{m_{P}} \varepsilon^{\mu \alpha \gamma \beta} k_{\gamma} p_{\beta}+\left[\frac{F_{A}}{m_{P}}+\frac{f_{P}}{p \cdot k}\right]\right. \\
& \left.\times\left(p \cdot k g^{\mu \alpha}-p^{\mu} k^{\alpha}\right)+\frac{f_{P}}{p \cdot k} p^{\mu} p^{\alpha}\right\} .
\end{aligned}
$$

Once the decay constant $f_{P}$ and the two SD axial and vector form factors $F_{A}$ and $F_{V}$ are known, the radiative decay rate can be calculated by using the formulas given in Appendix A. These formulas are expressed in terms of the convenient dimensionless variable

$$
x_{\gamma}=\frac{2 p \cdot k}{m_{P}^{2}} \quad \text { with } \quad 0 \leq x_{\gamma} \leq 1-\frac{m_{\ell}^{2}}{m_{P}^{2}},
$$

where $m_{\ell}$ is the mass of the outgoing lepton in the $P \rightarrow$ $\ell \bar{\nu}_{\ell} \gamma$ decay.

Our definition of the form factor $F_{A}$ differs from the definition, $F_{A}^{\mathrm{B}}$, of Refs. [21,23],

$$
F_{A}^{\mathrm{B}}=F_{A}+\frac{m_{P} f_{P}}{p \cdot k} .
$$

We note that $F_{A}^{\mathrm{B}}$ includes the pointlike infrared divergent contribution which totally dominates at low values of $x_{\gamma}$, thus obscuring the interesting structure-dependent contribution. For this reason, we strongly advocate the use of our definition [10]. Moreover, the sign of $F_{V}$ used in this paper is opposite to the one used in Ref. [21].

\section{FORM FACTORS FROM EUCLIDEAN CORRELATION FUNCTIONS}

In order to relate the hadronic matrix element to Euclidean correlation functions, the primary quantities computed in lattice calculations, it is useful to express the $H_{W}^{\alpha r}(k, \boldsymbol{p})$, defined in Eq. (1) in Minkowski space in terms of the contributions coming from the different time orderings. To this end, we define

$$
\begin{aligned}
H_{W}^{\alpha r}(k, \boldsymbol{p}) & =H_{W, 1}^{\alpha r}(k, \boldsymbol{p})+H_{W, 2}^{\alpha r}(k, \boldsymbol{p}), \\
j^{r}(\boldsymbol{k}) & =\epsilon_{\mu}^{r}(\boldsymbol{k}) \int d^{3} y e^{-i \boldsymbol{k} \cdot \boldsymbol{y}} j_{\mathrm{em}}^{\mu}(0, \boldsymbol{y})
\end{aligned}
$$


and perform the $t_{y}$ integral,

$$
\begin{aligned}
H_{W, 1}^{\alpha r}(k, \boldsymbol{p}) & =\int_{-\infty}^{0} d t_{y} e^{i E_{\gamma} t_{y}}\left\langle 0\left|j_{W}^{\alpha}(0) e^{i(\hat{H}-E-i \varepsilon) t_{y}} j^{r}(\boldsymbol{k})\right| P(\boldsymbol{p})\right\rangle \\
& =-i\left\langle 0\left|j_{W}^{\alpha}(0) \frac{1}{\hat{H}+E_{\gamma}-E-i \varepsilon} j^{r}(\boldsymbol{k})\right| P(\boldsymbol{p})\right\rangle, \\
H_{W, 2}^{\alpha r}(k, \boldsymbol{p}) & =\int_{0}^{\infty} d t_{y} e^{i E_{\gamma} t_{y}}\left\langle 0\left|j^{r}(\boldsymbol{k}) e^{-i(\hat{H}-i \varepsilon) t_{y}} j_{W}^{\alpha}(0)\right| P(\boldsymbol{p})\right\rangle \\
& =-i\left\langle 0\left|j^{r}(\boldsymbol{k}) \frac{1}{\hat{H}-E_{\gamma}-i \varepsilon} j_{W}^{\alpha}(0)\right| P(\boldsymbol{p})\right\rangle,
\end{aligned}
$$

where $\hat{H}$ is the QCD Hamiltonian operator, $E=$ $\sqrt{m_{P}^{2}+\boldsymbol{p}^{2}}$ is the energy of the decaying meson $P$, and $E_{\gamma}=|\boldsymbol{k}|$ is the energy of the outgoing real photon.

The important observation that makes the lattice calculation possible by using standard effective-mass/residue techniques is that the integrals over $t_{y}$ appearing in the definition of $H_{W}^{\alpha r}(k, \boldsymbol{p})$ can be Wick rotated to the Euclidean space without encountering any obstruction. Such obstructions arise whenever there are states propagating between the operators in the T-products that have energies smaller than the energy of the external states [24]. This does not happen in our case. For this reason, $H_{W, 1,2}^{\alpha r}(k, \boldsymbol{p})$ can be rewritten in terms of Euclidean integrals,

$H_{W, 1}^{\alpha r}(k, \boldsymbol{p})=-i \int_{-\infty}^{0} d t_{y}\left\langle 0\left|j_{W}^{\alpha}(0) e^{\left(\hat{H}+E_{y}-E\right) t_{y}} j^{r}(\boldsymbol{k})\right| P(\boldsymbol{p})\right\rangle$
$H_{W, 2}^{\alpha r}(k, \boldsymbol{p})=-i \int_{0}^{\infty} d t_{y}\left\langle 0\left|j^{r}(\boldsymbol{k}) e^{-\left(\hat{H}-E_{\gamma}\right) t_{y}} j_{W}^{\alpha}(0)\right| P(\boldsymbol{p})\right\rangle$,

both of which are convergent for physical (nonvanishing) photon energies. In Eq. (11) and below, $t_{y}$ is a Euclidean time variable and $r$ refers to the photon polarization as in Eq. (9). Indeed, the hadronic state of lowest energy that can propagate between the two currents is the pseudoscalar meson with spatial momentum $\boldsymbol{p}-\boldsymbol{k}$ (it appears in the timeordering $H_{W, 1}^{\alpha r}$ ) and we have

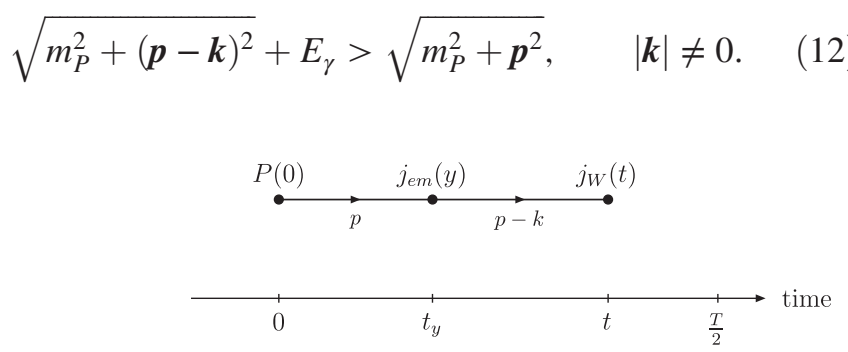

As a consequence, $H_{W}^{\alpha r}(k, \boldsymbol{p})$ can be rewritten as

$$
H_{W}^{\alpha r}(k, \boldsymbol{p})=-i \int d^{4} y e^{E_{\gamma} t_{y}-i \boldsymbol{k} \cdot \boldsymbol{y}} \epsilon_{\mu}^{r}(\boldsymbol{k}) \mathrm{T}\left\langle 0\left|j_{W}^{\alpha}(0) j_{\mathrm{em}}^{\mu}(y)\right| P(\boldsymbol{p})\right\rangle .
$$

From this observation, it follows that the hadronic matrix element can be extracted from the Euclidean correlation functions

$$
\begin{aligned}
C_{W}^{\alpha r}(t ; \boldsymbol{k}, \boldsymbol{p})= & -i \epsilon_{\mu}^{r}(\boldsymbol{k}) \int d^{4} y d^{3} \boldsymbol{x} e^{t_{y} E_{\gamma}-i \boldsymbol{k} \cdot \boldsymbol{y}+i \boldsymbol{p} \cdot \boldsymbol{x}} \\
& \times \mathrm{T}\left\langle 0\left|j_{W}^{\alpha}(t) j_{\mathrm{em}}^{\mu}(y) P(0, \boldsymbol{x})\right| 0\right\rangle,
\end{aligned}
$$

where $P=i \bar{\psi}_{D} \gamma_{5} \psi_{U}$ is a Hermitian pseudoscalar interpolating operator having the flavor quantum numbers of the incoming meson. In Eq. (14), using the translational invariance of the correlation function, we have moved the origin in time to the pseudoscalar source, $P(0, \boldsymbol{x})$, and placed the weak current at $t$. In the large- $t$ limit, one has

$$
\begin{aligned}
R_{W}^{\alpha r}(t ; \boldsymbol{k}, \boldsymbol{p}) & =\frac{2 E}{e^{-t\left(E-E_{\gamma}\right)}\langle P(\boldsymbol{p})|P(0)| 0\rangle} C_{W}^{\alpha r}(t ; \boldsymbol{k}, \boldsymbol{p}) \\
& =H_{W}^{\alpha r}(k, \boldsymbol{p})+\cdots,
\end{aligned}
$$

where the ellipsis represents the subleading exponentials.

The expressions for the correlation function $C_{W}^{\alpha r}(t ; \boldsymbol{k}, \boldsymbol{p})$ in Eq. (14) and for the ratio $R_{W}^{\alpha r}(t ; \boldsymbol{k}, \boldsymbol{p})$ in Eq. (15) refer to the ideal case of a lattice with infinite-time extent. The extraction of the matrix elements from correlation functions computed on a finite lattice in our numerical simulations is discussed in Appendix B. Although some of the details of the appendix refer to our specific lattice procedures (the choice of lattice fermions, renormalization of the operators, etc.), the strategy itself is general and can be directly translated to other lattice discretizations of QCD and of QED. Here in the main text, we use Fig. 2 to illustrate the strategy used in our numerical simulations, performed with (anti-) periodic boundary conditions in time for the (fermionic) bosonic fields, to extract the form factors. The two panels in Fig. 2 represent the forward $(0 \ll t \ll T / 2)$ and backward $(T / 2 \ll t \ll T)$ halves of the lattice. In both
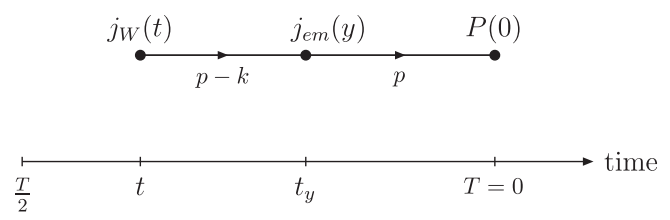

FIG. 2. Schematic diagrams representing the correlation function $\mathcal{C}_{W}^{\alpha r}(t, T / 2 ; \boldsymbol{k}, \boldsymbol{p})$ used to extract the form factors; see Appendix B. The interpolating operator for the meson $P$ and the weak current $j_{W}$ are placed at fixed times 0 and $t$, and the electromagnetic current $j_{\text {em }}$ is inserted at $t_{y}$ which is integrated over $0 \leq t_{y} \leq T$, where $T$ is the temporal extent of the lattice. The left and right panels correspond to the leading contributions to the correlation functions for $t_{y}<T / 2$ and $t_{y}>T / 2$, respectively, with mesons propagating with momenta $p$ or $p-k$. 

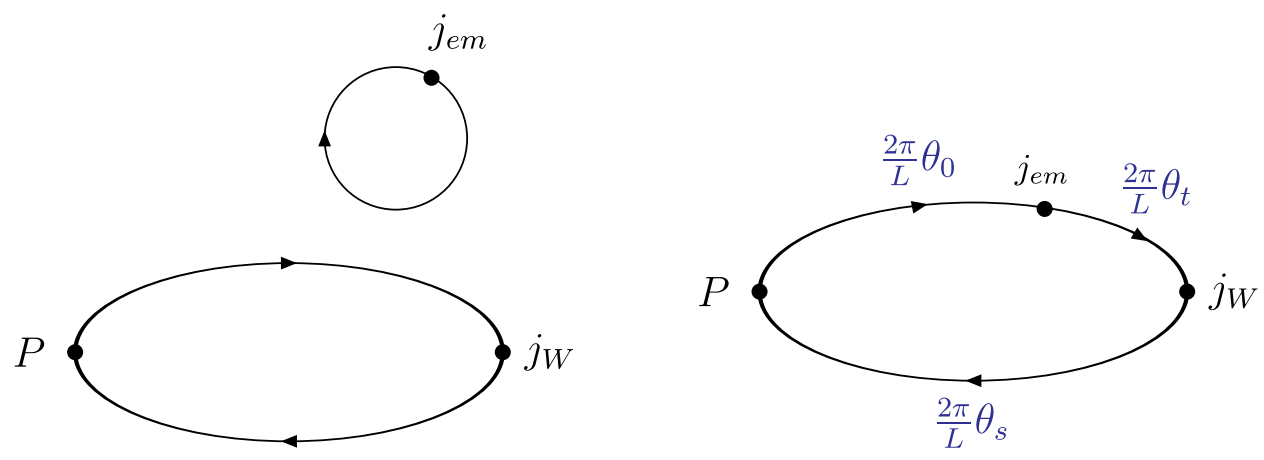

FIG. 3. The diagram on the left represents the contributions to the correlation functions arising from the emission of the photon by the sea quarks. In our numerical simulations, we work in the electroquenched approximation and neglect such diagrams. The diagram on the right explains our choice of the spatial boundary conditions, which allows us to set arbitrary values for the meson and photon spatial momenta. The spatial momenta of the valence quarks, modulo $2 \pi / L$, in terms of the twisting angles are as indicated. Each diagram implicitly includes all orders in QCD.

cases, the $t_{y}$ integral is dominated by the region in which $t_{y}$ is close to $t$, allowing for the propagation of the lightest state over the longest time interval.

In Fig. 3, we show two more diagrams to illustrate two important points concerning our numerical calculation of the correlation functions and of the form factors. The diagram in the left panel shows a quark-disconnected contribution to the correlation function originating from the possibility that the external real photon is emitted from sea quarks. In this work, we have been using the so-called electroquenched approximation in which the sea quarks are electrically neutral. In practice, this means that we have neglected the contributions represented by the diagram in the left panel of Fig. 3.

The quark-connected diagram in the right panel of Fig. 3 is shown in order to explain the strategy we have used to set the values of the spatial momenta. We exploited the fact that, by working within the electroquenched approximation, that is, in the absence of the contributions illustrated in the left panel of the figure, it is possible to choose arbitrary values of the spatial momenta by using different spatial boundary conditions for the quark fields [25]. More precisely, we set the boundary conditions for the "spectator" quark such that $\psi(x+\boldsymbol{n} L)=$ $\exp \left(2 \pi i n \cdot \boldsymbol{\theta}_{s}\right) \psi(x)$, where $L$ is the spatial extent of the lattice in each spatial direction. We treat the two propagators that are connected to the electromagnetic current as the result of the Wick contractions of two different fields having the same mass and electric charge but satisfying different boundary conditions [26]. This is possible at the price of accepting tiny violations of unitarity that are exponentially suppressed with the volume. By setting the boundary conditions as illustrated in the figure, we have thus been able to choose arbitrary (nonquantized) values for the meson and photon spatial momenta,

$$
\boldsymbol{p}=\frac{2 \pi}{L}\left(\boldsymbol{\theta}_{0}-\boldsymbol{\theta}_{s}\right), \quad \boldsymbol{k}=\frac{2 \pi}{L}\left(\boldsymbol{\theta}_{0}-\boldsymbol{\theta}_{t}\right)
$$

by tuning the real three-vectors $\boldsymbol{\theta}_{0, t, s}$. We find that the most precise results are obtained with small values of $|\boldsymbol{p}|$ and in particular with $\boldsymbol{p}=\mathbf{0}$.

The numerical results presented in the following sections have been obtained by setting the nonzero components of the spatial momenta along the third direction, that is,

$$
\boldsymbol{p}=(0,0,|\boldsymbol{p}|), \quad \boldsymbol{k}=\left(0,0, E_{\gamma}\right) .
$$

With this particular choice of the kinematical configuration, a convenient basis for the polarization vectors of the photon (see Appendix B for more details) is the one in which the two physical polarization vectors are given by

$\epsilon_{\mu}^{1}=\left(0,-\frac{1}{\sqrt{2}},-\frac{1}{\sqrt{2}}, 0\right), \quad \epsilon_{\mu}^{2}=\left(0, \frac{1}{\sqrt{2}},-\frac{1}{\sqrt{2}}, 0\right)$,

while the unphysical polarization vectors vanish identically, $\epsilon_{\mu}^{0}=\epsilon_{\mu}^{3}=0$. Notice that in this basis we have

$$
\epsilon^{r} \cdot p=\epsilon^{r} \cdot k=0
$$

and, consequently,

$$
\begin{aligned}
H_{A}^{j r}(k, \boldsymbol{p}) & =\frac{\epsilon_{j}^{r} m_{P}}{2} x_{\gamma}\left[F_{A}+\frac{2 f_{P}}{m_{P} x_{\gamma}}\right], \\
H_{V}^{j r}(k, \boldsymbol{p}) & =\frac{i\left(E_{\gamma} \boldsymbol{\epsilon}^{r} \wedge \boldsymbol{p}-E \boldsymbol{\epsilon}^{r} \wedge \boldsymbol{k}\right)^{j}}{m_{P}} F_{V} .
\end{aligned}
$$

Using these formulas, we have built the following numerical estimators:

$$
R_{A}(t)=\frac{1}{2 m_{P}} \sum_{r=1,2} \sum_{j=1,2} \frac{R_{A}^{j r}(t, T / 2 ; \boldsymbol{k}, \boldsymbol{p})}{\epsilon_{j}^{r}} \rightarrow x_{\gamma} F_{A}\left(x_{\gamma}\right)+\frac{2 f_{P}}{m_{P}},
$$


$R_{V}(t)=\frac{m_{P}}{4} \sum_{r=1,2} \sum_{j=1,2} \frac{R_{V}^{j r}(t, T / 2 ; \boldsymbol{k}, \boldsymbol{p})}{i\left(E_{\gamma} \boldsymbol{\epsilon}^{r} \wedge \boldsymbol{p}-E \boldsymbol{\epsilon}^{r} \wedge \boldsymbol{k}\right)^{j}} \rightarrow F_{V}\left(x_{\gamma}\right)$

for the form factors, which we determine by fitting to the plateaux in the region $0 \ll t \ll T / 2$. The discussion here and below corresponds explicitly to the forward half of the lattice $(0 \ll t \ll T / 2)$. We combine the results with those from the backward half $(T / 2 \ll t \ll T)$ by exploiting timereversal symmetry as explained in Appendix B.

The ratios $R_{W}^{j r}(t, T / 2 ; \boldsymbol{k}, \boldsymbol{p})$ appearing in Eqs. (21) and (22), which we evaluate separately for the axial $(W=A)$ and vector $(W=V)$ components of the weak current, are the finite- $T$ generalizations [see Eq. (B19)] of the ratios $R_{W}^{\alpha r}(t ; \boldsymbol{k}, \boldsymbol{p})$ defined above in Eq. (15). The values of the meson energies and of the matrix elements $\langle P|P| 0\rangle$ needed to build these estimators have been obtained from standard effective-mass/residue analyses of pseudoscalar-pseudoscalar two-point functions. We have also computed the pseudoscalar-axial two-point functions from which we have extracted the decay constants $f_{P}$ on our data sets in order to be able to separate the SD axial form factor $F_{A}$ from the pointlike contribution $2 f_{P} /\left(m_{P} x_{\gamma}\right)$.

\section{NONPERTURBATIVE SUBTRACTION OF INFRARED DIVERGENT DISCRETIZATION EFFECTS}

In this section, we want to stress a very important issue associated with infrared divergent cutoff effects which can jeopardize the extraction of $F_{A}$ at small values of $x_{\gamma}$. We also introduce a strategy to overcome this problem.

In Fig. 4, we plot $F_{A}\left(x_{\gamma}\right)+2 f_{P} /\left(m_{P} x_{\gamma}\right)$, the sum of the pointlike and SD axial form factors which are extracted directly from the correlation functions using $R_{A}(t)$ [see Eq. (21)], as a function of $x_{\gamma}$ for the $K$ (left panel) and the $D_{s}$ (right panel) mesons. The pointlike contribution, $2 f_{P} /\left(m_{P} x_{\gamma}\right)$, dominates the axial form factor in the full physical range of photon energies and is overwhelming at small $x_{\gamma}$. Using the decay constant and mass, computed in the standard way from the two-point functions, we can in principle subtract the pointlike term and extract $F_{A}\left(x_{\gamma}\right)$. However, this turns out to be very difficult because of the possible presence of discretization effects which cannot be excluded by the WI of the lattice action. Moreover, these lattice artifacts diverge as $x_{\gamma} \rightarrow 0$. We now propose a nonperturbative method to eliminate this problem.

At finite lattice spacing, the axial form factor is constrained, as in the continuum [see Eq. (4)], by an exact lattice WI,

$\frac{2 \sin \left(k_{\mu} a / 2\right)}{a} H_{L}^{\alpha \mu}(k, \boldsymbol{p})=-\left\langle 0\left|j_{A}^{\alpha}(0)\right| P(\boldsymbol{p})\right\rangle=-f_{P}^{L} p_{L}^{\alpha}$, that is true at all orders in the lattice spacing $a$ (see Appendix C). The label $L$ here, and in the remainder of this section, stands for "lattice" as the discussion concerns the Ward identity in a discrete space-time. It should not be confused with the spatial extent of the lattice. This however does not exclude the presence of cutoff effects in Eq. (21). These are terms of $O\left(a^{2}\right)^{1}$ and, in particular, include contributions of $O\left(a^{2} / x_{\gamma}\right)$,

$$
\begin{aligned}
\frac{R_{A}(t)}{x_{\gamma}} \rightarrow & \frac{1}{4 x_{\gamma}} \sum_{r=1,2} \sum_{j=1,2} \frac{2 H_{A}^{j r}(k, \boldsymbol{p})}{\epsilon_{j}^{r} m_{P}}=\left[F_{A}\left(x_{\gamma}\right)+a^{2} \Delta F_{A}\left(x_{\gamma}\right)\right] \\
& +\frac{2}{m_{P} x_{\gamma}}\left(f_{P}+a^{2} \Delta f_{P}\right)+\cdots
\end{aligned}
$$

where the ellipsis represents higher orders in $a^{2}$, while the quantities $\Delta F_{A}$ and $\Delta f_{P}$ depend upon the parameters of the theory regularized on the lattice, on the light- and heavyquark masses and upon $\Lambda_{\mathrm{QCD}}$. Discretization effects in the pseudoscalar masses are also absorbed into $\Delta F_{A}$ and $\Delta f_{P}$. The crucial point to notice is that the lattice decay constant of the WI in Eq. (23) $f_{P}^{L} \neq f_{P}+a^{2} \Delta f_{P}$. This implies the presence of the extra term of $O\left(a^{2} / x_{\gamma}\right)$ which appears, in spite of the naive expectations based on the exact lattice WI. Thus, the coefficient of the last term in Eq. (24) is not in general given by $2 f_{P}^{L} /\left(m_{P} x_{\gamma}\right)$, where $f_{P}^{L}$ is the quantity extracted from the axial-pseudoscalar lattice correlation functions at finite lattice spacing. More precisely, once the matrix element $\left\langle 0\left|j_{A}^{\alpha}(0)\right| P(p)\right\rangle$ is parametrized as in Eq. (23), the definition of $f_{P}^{L}$ at fixed cutoff depends upon the choice of the index $\alpha$ and of the lattice momentum $p_{L}^{\alpha}$ and, for this reason, is not unique. Therefore, given a generic definition of $f_{P}^{L}$, one cannot expect a complete cancellation of the infrared divergent term on the right-hand side of Eq. (24), because a residual lattice artifact will survive,

$$
\begin{aligned}
F_{A}^{\mathrm{sub}}\left(x_{\gamma}\right) & =\frac{1}{4 x_{\gamma}} \sum_{r=1,2} \sum_{j=1,2} \frac{2 H_{A}^{j r}(k, \boldsymbol{p})}{\epsilon_{j}^{r} m_{P}}-\frac{2 f_{P}^{L}}{x_{\gamma} m_{P}} \\
& =F_{A}\left(x_{\gamma}\right)+a^{2} \Delta F_{A}\left(x_{\gamma}\right)+\frac{2 a^{2} \Delta \tilde{f}_{P}}{x_{\gamma} m_{P}}
\end{aligned}
$$

generating an effective, unphysical infrared divergent contribution to $F_{A}^{\mathrm{sub}}\left(x_{\gamma}\right)$ at finite cutoff $\left(a^{2} \Delta \tilde{f}_{P}=f_{P}-f_{P}^{L}+\right.$ $\left.a^{2} \Delta f_{P}\right)$. This phenomenon is illustrated for the $D_{s}$ meson in Fig. 5 where $F_{A}^{\text {sub }}$ is plotted as function of $x_{\gamma}$. Since the subtraction of the potentially divergent term is incomplete, we observe a fast rise of the effective $F_{A}^{\mathrm{sub}}\left(x_{\gamma}\right)$ at small

\footnotetext{
${ }^{1}$ We assume here that we are using a lattice discretization in which the leading artifacts are $O\left(a^{2}\right)$. For Wilson fermions in which they are $O(a)$, the discussion has to be modified accordingly.
} 

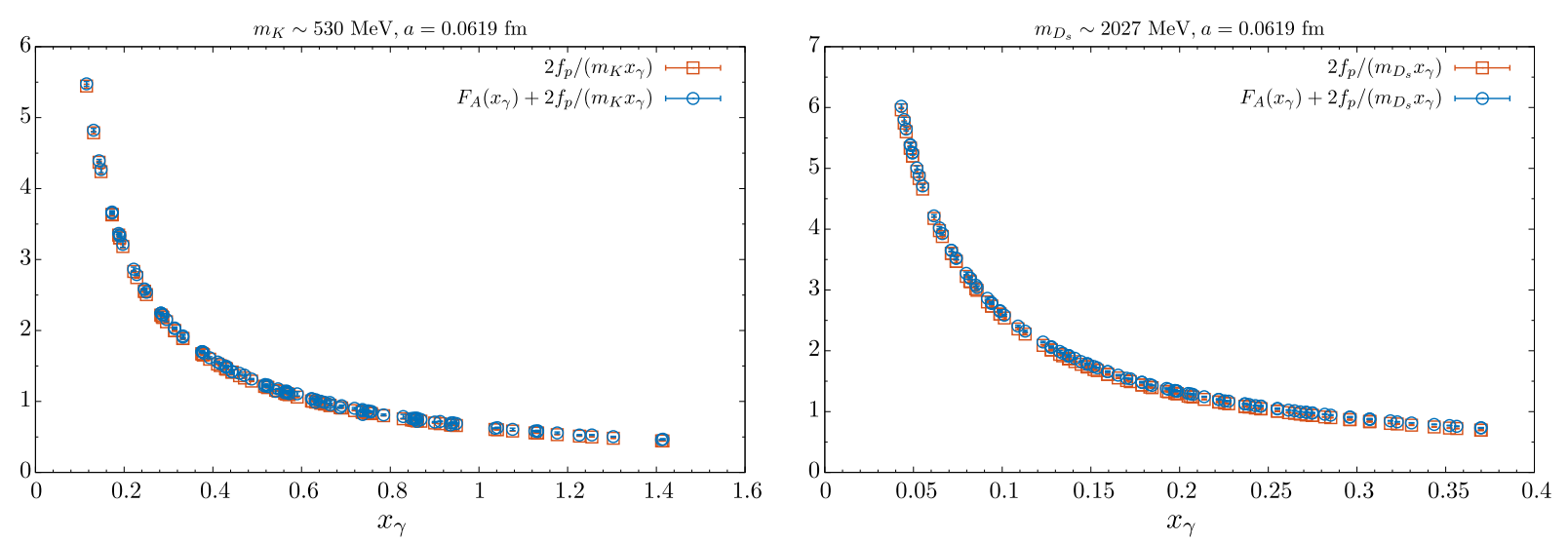

FIG. 4. The blue circles represent $F_{A}\left(x_{\gamma}\right)+2 f_{P} /\left(m_{P} x_{\gamma}\right)$, extracted directly from $R_{A}(t)$, as a function of $x_{\gamma}$ for the $K$ meson (left) and for the $D_{s}$ meson (right). The red squares represent the pointlike contribution given by $2 f_{P} /\left(m_{P} x_{\gamma}\right)$. The data are taken from the ensemble D15.48 of Ref. [15].

values of $x_{\gamma}$. For this reason, even if one has data at different values of the lattice spacing, it is particularly difficult to extract the continuum form factor $F_{A}\left(x_{\gamma}\right)$ from $F_{A}^{\text {sub }}\left(x_{\gamma}\right)$, especially at small $x_{\gamma}$ and for heavy mesons. This is illustrated by the intermediate (red) points in Fig. 5 which were obtained by fitting and subtracting the $O\left(a^{2} / x_{\gamma}\right)$ artifacts. The divergence at small $x_{\gamma}$ is reduced but the relative statistical uncertainties are increased.

We now present an alternative strategy that avoids this problem. In Appendix $\mathrm{C}$, we show that the correlation function $C_{A}^{\alpha r}(t ; \boldsymbol{k}, \boldsymbol{p})$ has a smooth behavior as a function of $\boldsymbol{k}$ and that from $C_{A}^{\alpha r}(t ; \mathbf{0}, \boldsymbol{p})$ it is possible to extract directly $H_{A}^{i r}(0, \boldsymbol{p})=\epsilon_{i}^{r} f_{P}$ [see Eq. (20)]. We can then construct the quantity

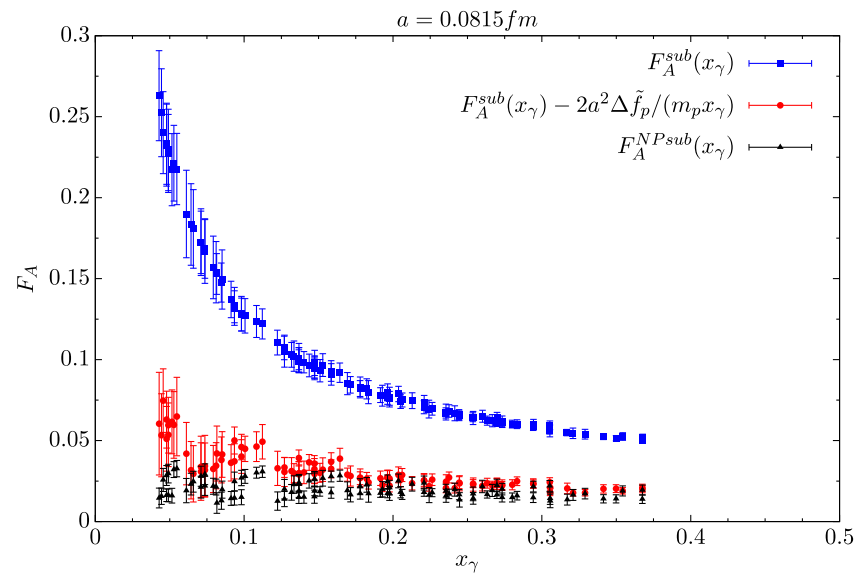

FIG. 5. Study of $F_{A}$ for the $D_{s}$ meson. The upper (blue) points show $F_{A}^{\mathrm{sub}}\left(x_{\gamma}\right)$ obtained from Eq. (25). The divergence at small $x_{\gamma}$ is reduced by fitting and subtracting the $O\left(a^{2} / x_{\gamma}\right)$ artifacts, at the price of increased uncertainties at small $x_{\gamma}$; these are the intermediate (red) points. The most accurate results are given by $F_{A}^{\mathrm{NPsub}}$, obtained by the nonperturbative subtraction of these artifacts as in Eq. (27) and are shown by the lower (black) points. The data are obtained using the ensemble B55.32 of Ref. [15].

$$
\bar{R}_{A}(t)=e^{-t E_{\gamma}} \frac{\sum_{r=1,2} \sum_{j=1,2} \frac{C_{A}^{j r}(t, T / 2 ; \boldsymbol{k}, \boldsymbol{p})}{\epsilon_{j}^{r}}}{\sum_{r=1,2} \sum_{j=1,2} \frac{C_{A}^{j r}(t, T / 2 ; \mathbf{0}, \boldsymbol{p})}{\epsilon_{j}^{r}}}-1
$$

that, by construction, vanishes identically at $x_{\gamma}=0$. Up to statistical uncertainties, each term in the sums in the numerator and denominator of Eq. (26) is independent of the indices $j, r$. For the study of the constraints imposed by the electromagnetic Ward identity, it is helpful to view the right-hand side as $H_{A}^{j r}(k, p) / H_{A}^{j r}(0, p)-1$ (which is also independent of $j, r$ ). From the improved estimator $\bar{R}_{A}(t)$, we can extract the structure-dependent form factor $F_{A}$ using

$$
\frac{2 f_{P}}{m_{P} x_{\gamma}} \bar{R}_{A}(t) \rightarrow F_{A}^{\mathrm{NPsub}}\left(x_{\gamma}\right)=F_{A}\left(x_{\gamma}\right)+O\left(a^{2}\right),
$$

a quantity that we also show in Fig. 5 and that, in contrast to $F_{A}^{\text {sub }}$, does not show any divergent behavior at small $x_{\gamma}$. The reduction of the uncertainty on $F_{A}\left(x_{\gamma}\right)$ using $\bar{R}_{A}(t)$, with respect to a fit to the right-hand side of Eq. (25), as shown in Fig. 5, is impressive, particularly at small $x_{\gamma}$ and also for heavy mesons where there are discretization effects of $O\left(a^{2} m_{D_{(s)}}^{2}\right)$. In the following, we will only present results obtained with this method.

The knowledge of $C_{A}^{j r}(t, T / 2 ; \mathbf{0}, \boldsymbol{p})$ allows us also to define an alternative estimator for the form factor $F_{V}\left(x_{\gamma}\right)$, namely,

$$
\begin{aligned}
\bar{R}_{V}(t) & =f_{P} m_{P} \frac{\left(\sum_{r=1,2} \sum_{j=1,2} \frac{C_{V}^{j r}(t, T / 2 ; \boldsymbol{k}, \boldsymbol{p})-C_{V}^{j r}(t, T / 2 ; \mathbf{0}, \boldsymbol{p})}{i\left(E_{\gamma} r^{r} \wedge \boldsymbol{p}-E \boldsymbol{\epsilon}^{r} \wedge \boldsymbol{k}\right)^{j}} e^{-t E_{\gamma}}\right)}{\left(\sum_{r=1,2} \sum_{j=1,2} \frac{C_{A}^{j r}(t, T / 2 ; \mathbf{0}, \boldsymbol{p})}{\epsilon_{j}^{r}}\right)} \\
& \rightarrow F_{V}\left(x_{\gamma}\right),
\end{aligned}
$$

that we find has reduced statistical errors compared to $R_{V}(t)$. Note that because of parity symmetry the correlation 
function $C_{V}^{j r}(t, T / 2 ; \mathbf{0}, \boldsymbol{p})=0$, but this is only approximately true when it is estimated using a finite statistical sample. We find that taking the difference $C_{V}^{j r}(t, T / 2 ; \boldsymbol{k}, \boldsymbol{p})-$ $C_{V}^{j r}(t, T / 2 ; \mathbf{0}, \boldsymbol{p})$ in the numerator of Eq. (28) results in a significant reduction of the statistical uncertainty for physical values of $x_{\gamma}$ [see Eq. (7)].

\section{NUMERICAL RESULTS}

The results presented in this paper were obtained using the ETMC gauge ensembles with $N_{f}=2+1+1$ dynamical quarks at three different values of the lattice spacing, $a=0.0885(36), 0.0815(30)$, and $0.0619(18) \mathrm{fm}$, with meson masses in the range 220-2110 MeV. Details about these ensembles are given in Table II of Ref. [15]; see also Table I in Appendix D. In total, we have included 125 different combinations of momenta obtained by assigning to each of the $\theta_{i=0, t, s}$ five different values; making the same assignments for all choices of the quark masses. In the figures below, we illustrate the quality and features of our results by showing examples of plots for light and heavy mesons. The plots used for illustration correspond to unphysical values of the $\overline{\mathrm{MS}}$ renormalized light-quark mass, $m_{u d}(2 \mathrm{GeV})=11.7 \mathrm{MeV}$. The corresponding meson masses are $m_{D_{s}}=2027(3) \mathrm{MeV}, m_{D}=1929(6) \mathrm{MeV}$, $m_{K}=530(2) \mathrm{MeV}$, and $m_{\pi}=228(2) \mathrm{MeV}$. Similar plots can be shown for other values of the simulation parameters.

The scale setting is taken from Ref. [19], where the continuum value of $r_{0}$ [27] was obtained imposing $m_{\pi}^{\exp }=m_{\pi^{0}}=134.98 \mathrm{MeV}$ and $f_{\pi}^{\exp }=130.41(20) \mathrm{MeV}$. The values of the strange and charm quark masses, obtained by extrapolating the kaon and $D$ meson masses
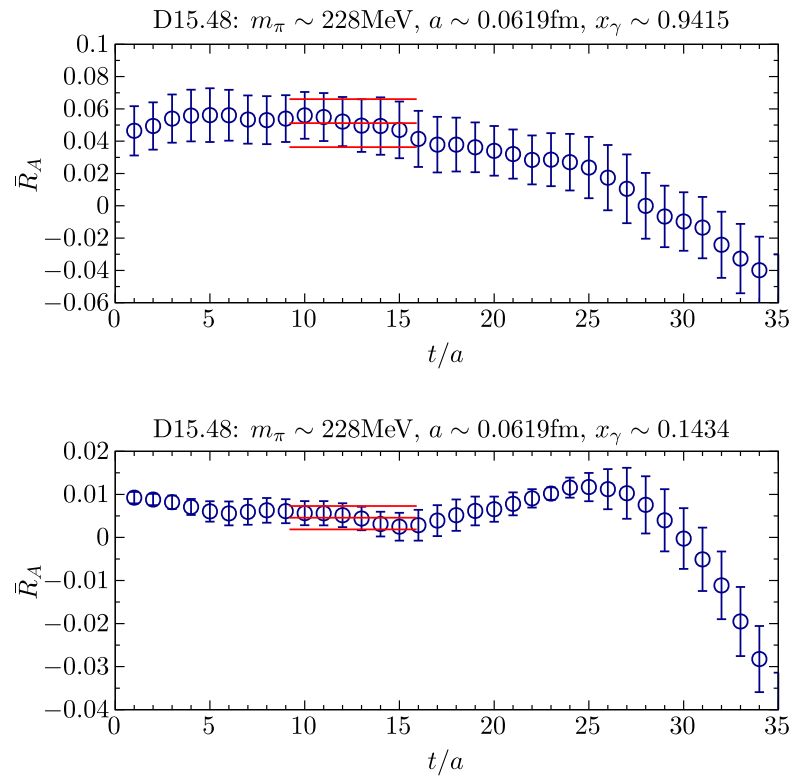

to the continuum and at the physical point in the light-quark masses, are $m_{s}(2 \mathrm{GeV})=99.6(4.3) \mathrm{MeV}$ and $m_{c}(2 \mathrm{GeV})=1.176(39) \mathrm{GeV}$. In the following for the renormalized quark mass, we shall use $m=\mu / Z_{P}$, where $\mu$ is the twisted mass of the given quark and $Z_{P}$ is the renormalization constant of the pseudoscalar density in the $\overline{\mathrm{MS}}$ scheme, at $2 \mathrm{GeV}$, computed with method M2 [19]. The values of $\mu$ used in our simulation can also be found in Tables I and II in Appendix D (see also Table II of Ref. [15]). Renormalization of the corresponding axialvector and vector currents with twisted mass fermions gives $F_{A}=Z_{V} F_{A}^{0}$ and $F_{V}=Z_{A} F_{V}^{0}$, where $F_{A}^{0}$ and $F_{V}^{0}$ are the unrenormalized quantities as explained in Eq. (B6), $Z_{A}$ has been computed with method M2 and $Z_{V}$ with the WI [19]. In Table II of Appendix D, we give further details of our simulation including the values of the angles $\theta_{i=0, s, t}$ used to fix the hadron and photon momenta; see Eq. (16).

In Figs. 6 and 7, we show examples of plateaux for the ratios $\bar{R}_{A, V}(t)$, defined in Eqs. (26) and (28), respectively, for $K$ and $D$ mesons. These figures are representative of the signal quality also for other values of masses, momenta, and lattice spacings. The values of all the form factors discussed in the following have been extracted from the plateaux obtained by using Eqs. (26) and (28). The time interval used for the extraction has been chosen, for each of the data ensembles and for each of the mesons, in such a way as to observe a reasonable plateau for all values of the meson/photon momenta. Regarding the quality of the plateaux, we have performed an extensive study of the dependence of the results on the time interval chosen for the fits and the differences are included in the estimates of the uncertainties on the extracted values of $F_{A, V}$.
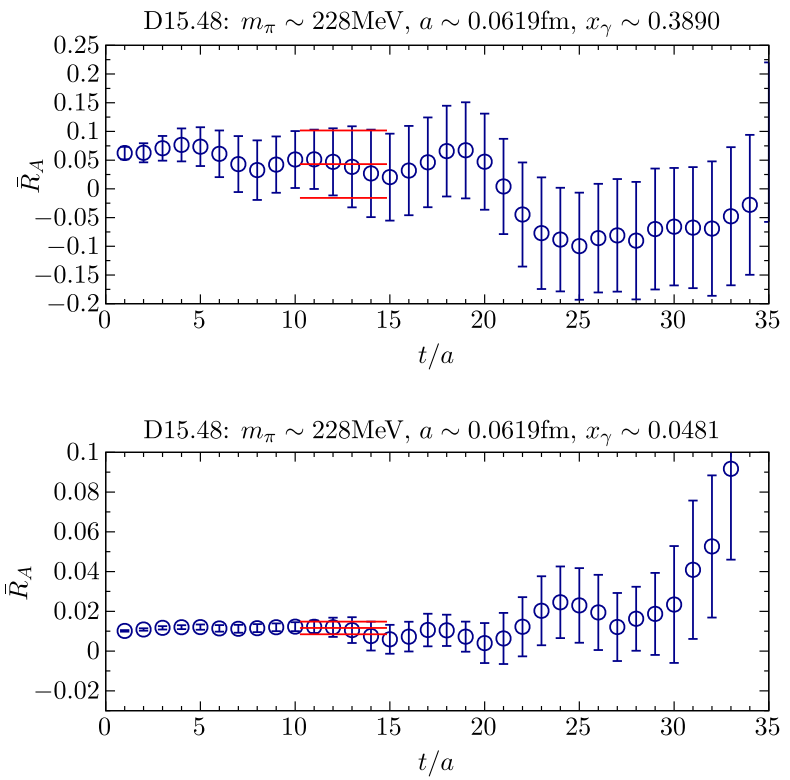

FIG. 6. Examples of fits to plateaux for the ratio $\bar{R}_{A}(t)$ for the kaon (left) and $D$ meson (right) at larger (upper panels) or smaller (lower panels) values of $x_{\gamma}$. The values obtained from the fits, together with their uncertainties, are indicated by the horizontal (red) bands. 

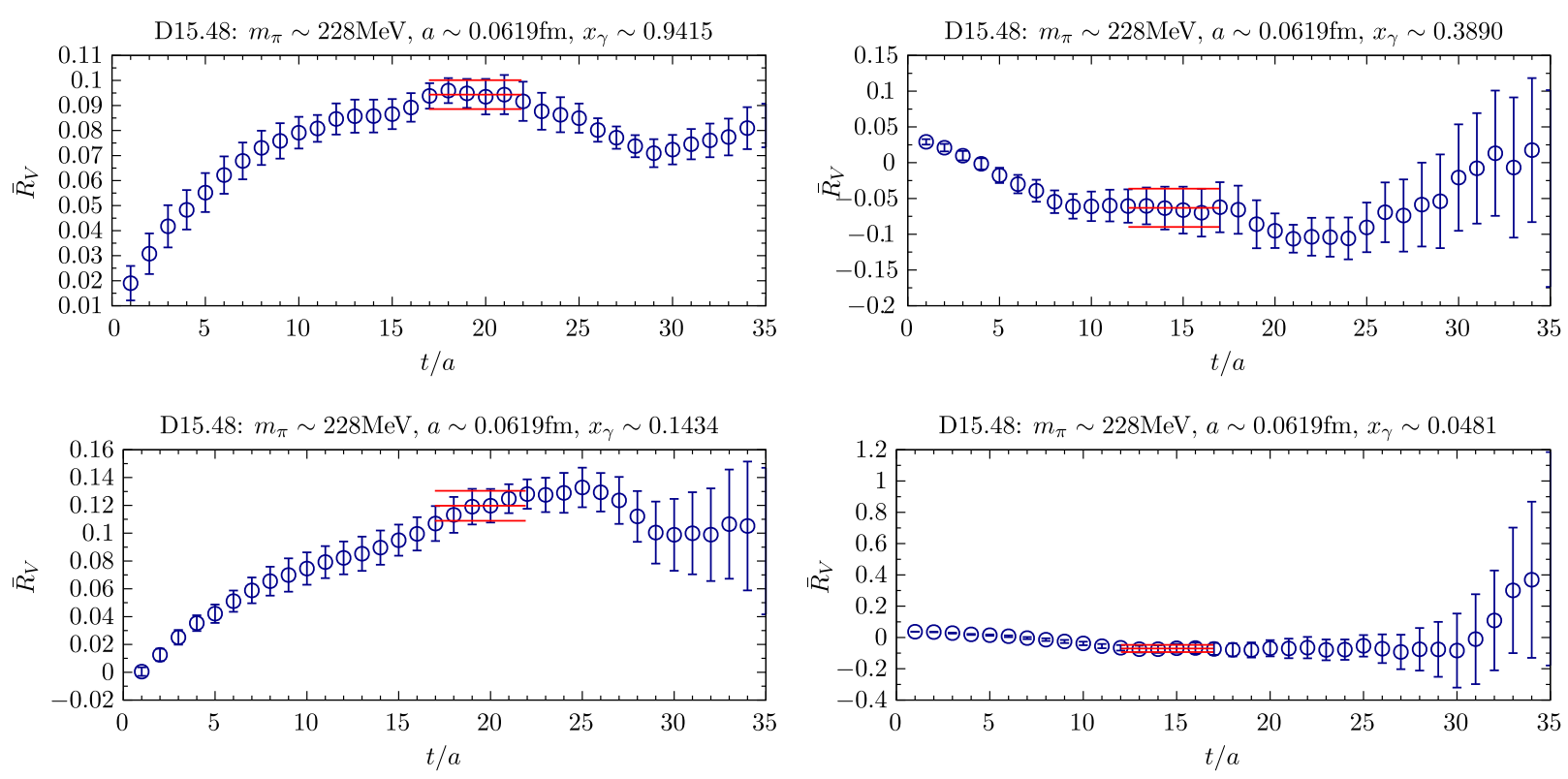

FIG. 7. Examples of fits to plateaux for the ratio $\bar{R}_{V}(t)$ for the kaon (left) and $D$ meson (right) at larger (upper panels) or smaller (lower panels) values of $x_{\gamma}$. The values obtained from the fits, together with their uncertainties, are indicated by the horizontal (red) bands.

In order to extract the form factors $F_{A, V}$ at physical values of the quark masses and in the continuum limit, we have used a variety of fitting formulas for light and heavy mesons as discussed below.
For pions and kaons, we have covered the full physical range of $x_{\gamma}, 0 \leq x_{\gamma} \leq 1-m_{\ell}^{2} / m_{\pi, K}^{2}$ (indeed we even have data for unphysical values corresponding to $x_{\gamma}>1$ ).

For the pion, guided by ChPT, we fit to the formula

$$
F_{A, V}\left(x_{\gamma}\right)=\frac{m_{\pi}}{f_{\pi}}\left[\left(c_{0}+c_{0}^{\prime} \frac{m_{\pi}^{2}}{\left(4 \pi f_{\pi}\right)^{2}}+\tilde{c}_{0} \frac{a^{2}}{r_{0}^{2}}\right)+\left(c_{1}^{\prime} \frac{m_{\pi}^{2}}{\left(4 \pi f_{\pi}\right)^{2}}+\tilde{c}_{1} \frac{a^{2}}{r_{0}^{2}}\right) x_{\gamma}\right]
$$

This is certainly not the most general formula to include higher-order terms in ChPT; for example, it does not contain chiral logarithms, but it is sufficiently simple and adequate to describe the pion data. The two coefficients $\tilde{c}_{0}$ and $\tilde{c}_{1}$ take into account possible mass-independent discretization effects. $c_{1}^{\prime}$ is multiplied by $m_{\pi}^{2}$ because it arises in higher orders in ChPT. On the other hand, the discretization term proportional to $\tilde{c}_{1}$ is not multiplied by the mass of the meson because at this order in $a$ there is an explicit violation of chiral invariance in the lattice fermion Lagrangian.

When using the simpler expression in Eq. (29), we exclude data at pion masses $m_{\pi} \gtrsim 350 \mathrm{MeV}$. Since in our data we have pion masses up to about $500 \mathrm{MeV}$, we have also performed fits in the full range by modifying Eq. (29) to include higher-order terms as follows:

$$
\begin{aligned}
F_{A, V}\left(x_{\gamma}\right)= & \frac{m_{\pi}}{f_{\pi}}\left[\left(c_{0}+c_{0}^{\prime} \frac{m_{\pi}^{2}}{\left(4 \pi f_{\pi}\right)^{2}}+\tilde{c}_{0} \frac{a^{2}}{r_{0}^{2}}+\Delta c_{0}^{\prime} \frac{m_{\pi}^{4}}{\left(4 \pi f_{\pi}\right)^{4}}+\Delta \tilde{c}_{0} a^{2} m_{\pi}^{2}\right)\right. \\
& \left.+\left(c_{1}^{\prime} \frac{m_{\pi}^{2}}{\left(4 \pi f_{\pi}\right)^{2}}+\tilde{c}_{1} \frac{a^{2}}{r_{0}^{2}}+\Delta c_{1}^{\prime} \frac{m_{\pi}^{4}}{\left(4 \pi f_{\pi}\right)^{4}}+\Delta \tilde{c}_{1} a^{2} m_{\pi}^{2}\right) x_{\gamma}\right] .
\end{aligned}
$$

The higher-order coefficients $\Delta c_{0}^{\prime}, \Delta \tilde{c}_{0}, \tilde{c}_{1}, \Delta c_{1}^{\prime}$, and $\Delta \tilde{c}_{1}$ have very little effect on the extrapolated results and for this reason they are not well determined. Indeed, they only contribute to a slight increase in the uncertainty in the value of the pion form factors at $x_{\gamma}=0$ and in the slope in $x_{\gamma}$.
Similarly, in the different fits that we performed, we also added some of the possible lattice artifacts that break Lorentz invariance, for example, those proportional to $a^{2}|\boldsymbol{k}|^{2}$ (in the frame where the meson is at rest), where $\boldsymbol{k}$ is the momentum of the photon. We found that their effect 

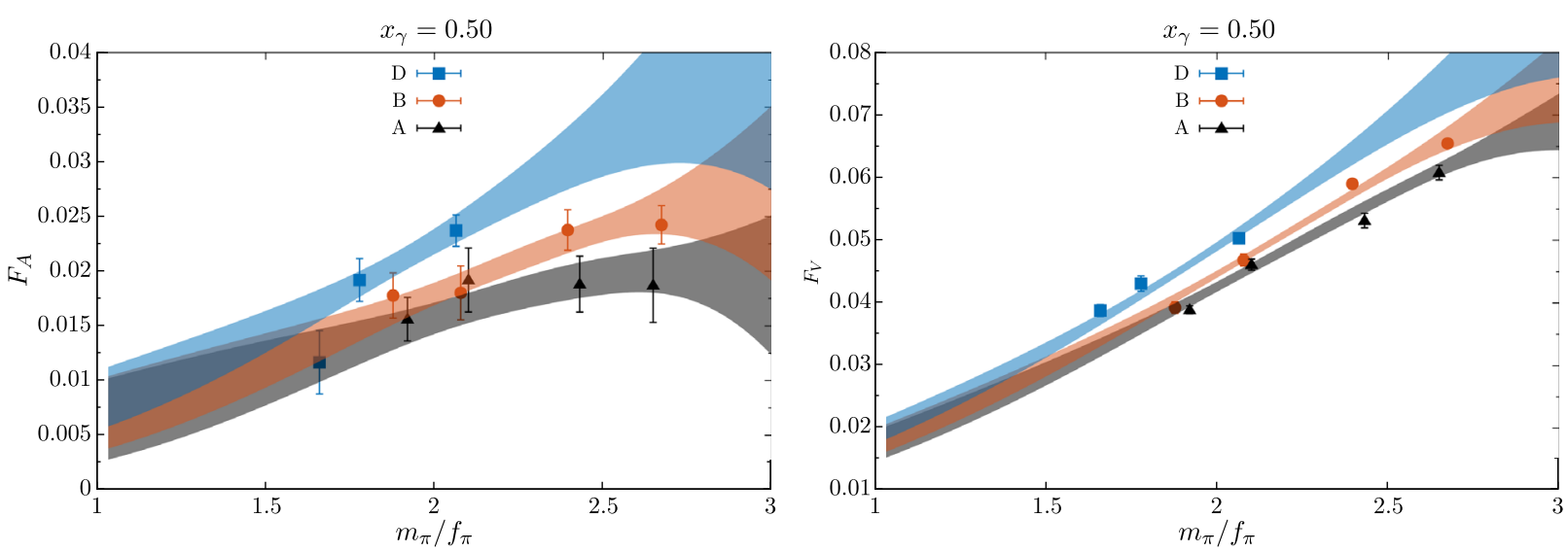

FIG. 8. Dependence of $F_{A}\left(x_{\gamma}\right)$ (left panel) and $F_{V}\left(x_{\gamma}\right)$ (right panel) for the pion on the light-quark masses, specifically on $m_{\pi} / f_{\pi}$. The letters A, B, and D refer to the sets of gauge field configurations at different lattice spacings listed in Table I of Appendix D.

is very small and this was only taken into account in the evaluation of the final uncertainties. Since $S U(3)$ breaking effects may be important, and we only have results obtained at two values of the strange quark mass, for the kaon we first interpolate the form factors to the physical kaon mass and then fit them to the formula

$$
F_{A, V}\left(x_{\gamma}\right)=\frac{m_{K}}{f_{K}}\left[\left(c_{0}+c_{0}^{\prime} \frac{m_{\pi}^{2}}{\left(4 \pi f_{\pi}\right)^{2}}+\tilde{c}_{0} \frac{a^{2}}{r_{0}^{2}}\right)+\left(c_{1}+c_{1}^{\prime} \frac{m_{\pi}^{2}}{\left(4 \pi f_{\pi}\right)^{2}}+\tilde{c}_{1} \frac{a^{2}}{r_{0}^{2}}\right) x_{\gamma}\right]
$$

with pion masses $m_{\pi}<350 \mathrm{MeV}$ and

$$
\begin{aligned}
F_{A, V}\left(x_{\gamma}\right)= & \frac{m_{K}}{f_{K}}\left[\left(c_{0}+c_{0}^{\prime} \frac{m_{\pi}^{2}}{\left(4 \pi f_{\pi}\right)^{2}}+\tilde{c}_{0} \frac{a^{2}}{r_{0}^{2}}+\Delta c_{0}^{\prime} \frac{m_{\pi}^{4}}{\left(4 \pi f_{\pi}\right)^{4}}+\Delta \tilde{c}_{0} a^{2} m_{\pi}^{2}\right)\right. \\
& \left.+\left(c_{1}+c_{1}^{\prime} \frac{m_{\pi}^{2}}{\left(4 \pi f_{\pi}\right)^{2}}+\tilde{c}_{1} \frac{a^{2}}{r_{0}^{2}}+\Delta c_{1}^{\prime} \frac{m_{\pi}^{4}}{\left(4 \pi f_{\pi}\right)^{4}}+\Delta \tilde{c}_{1} a^{2} m_{\pi}^{2}\right) x_{\gamma}\right]
\end{aligned}
$$

in the full range of pion masses. Formulas (31) and (32) for the kaon are equivalent to those in (29) and (30), respectively, for the pion. The presence of the constant term $c_{1}$ in Eqs. (31) and (32) is a reflection of the fact that the strange quark mass is fixed to its physical value. To simplify the notation, we have used the same symbols for the coefficients in Eqs. (29)-(32), but the reader should note that their values are different in each case. We do not have sufficient data to include terms proportional to $m_{K}^{2} m_{\pi}^{2}$ or $m_{\pi}^{4}$ with logarithmic corrections in Eq. (32). In order to show that the quark mass dependence is well described by our Ansatz, in Fig. 8 we plot the dependence of the pion's $F_{A}\left(x_{\gamma}=0.5\right)$ (left panel) and $F_{V}\left(x_{\gamma}=0.5\right)$ (right panel) on the light-quark masses (specifically on $m_{\pi} / f_{\pi}$ ) for different values of the lattice spacing. The results are compared to the fit to the data obtained using the expression in Eq. (30). Some interpolation of the data in $x_{\gamma}$ was needed to match the points and plot them as a function of $m_{\pi} / f_{\pi}$ only.

In Fig. 9, we present the values of the pion (left panels) and kaon (right panels) form factors $F_{A}\left(x_{\gamma}\right)$ (upper panels) and $F_{V}\left(x_{\gamma}\right)$ (lower panels) as a function of $x_{\gamma}$ for the configurations at $a=0.0619 \mathrm{fm}$. The plotted points with error bars correspond to different values of the light-quark mass at several values of $x_{\gamma}$. The points with large uncertainties $\left(\sigma_{F_{A, V}} \geq 0.01\right.$ for the kaon or $\sigma_{F_{A, V}} \geq 0.008$ for the pion) are shown with faint gray symbols. These points are obtained for mesons with substantial nonzero momenta $\boldsymbol{p} \neq \mathbf{0}$. The results of our simulation are compared to the lowest order in $\mathrm{ChPT}$, given by

$$
\begin{aligned}
& F_{A}\left(x_{\gamma}\right)=\text { const. }=\frac{8 m_{P}}{f_{P}}\left(L_{9}^{r}+L_{10}^{r}\right) \\
& F_{V}\left(x_{\gamma}\right)=\text { const. }=\frac{m_{P}}{4 \pi^{2} f_{P}},
\end{aligned}
$$

where $P$ represents $\pi$ or $K$ and we take $\left(L_{9}^{r}+L_{10}^{r}\right) \simeq$ 0.0017 [28]; this is indicated by the horizontal red lines. The blue lines and green bands are the results and uncertainties of the fits, obtained using Eqs. (30) and (32) after the extrapolation to physical quark masses and to zero lattice spacing has been performed. In Fig. 10, we show the value of the pion (left) and the kaon (right) form factors $F_{A}\left(x_{\gamma}\right)$ (upper) and $F_{V}\left(x_{\gamma}\right)$ (lower) as a function of $x_{\gamma}$, extrapolated to the continuum at the physical point, 

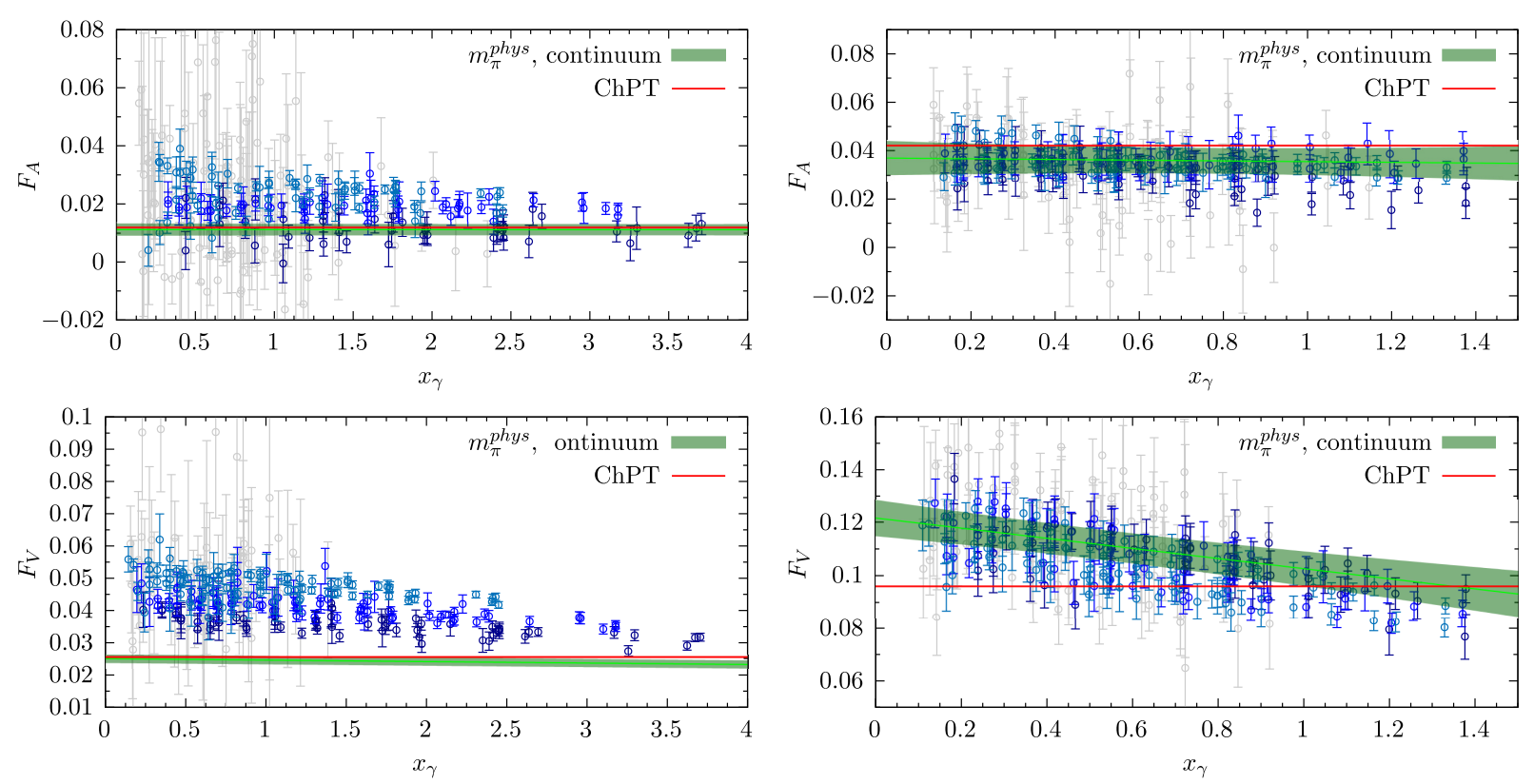

FIG. 9. Extracted values of the pion (left) and kaon (right) form factors $F_{A}\left(x_{\gamma}\right)$ (upper) and $F_{V}\left(x_{\gamma}\right)$ (lower) as a function of $x_{\gamma}$ for the configurations at $a=0.0619 \mathrm{fm}$. The horizontal red lines correspond to the lowest order ChPT prediction in Eq. (33). The green lines and bands are the results of the fits, using the formulas given in Eqs. (29) and (31), after extrapolation to the continuum limit and physical quark masses, together with the corresponding uncertainties.

either using Eqs. (29) and (31) for the pion and kaon, respectively, to fit the data, full green bands, or by using Eqs. (30) and (32), shaded blue bands. In the figure, we also show the values of the form factors for selected values of $x_{\gamma}$ extrapolated to the continuum and to the physical point,
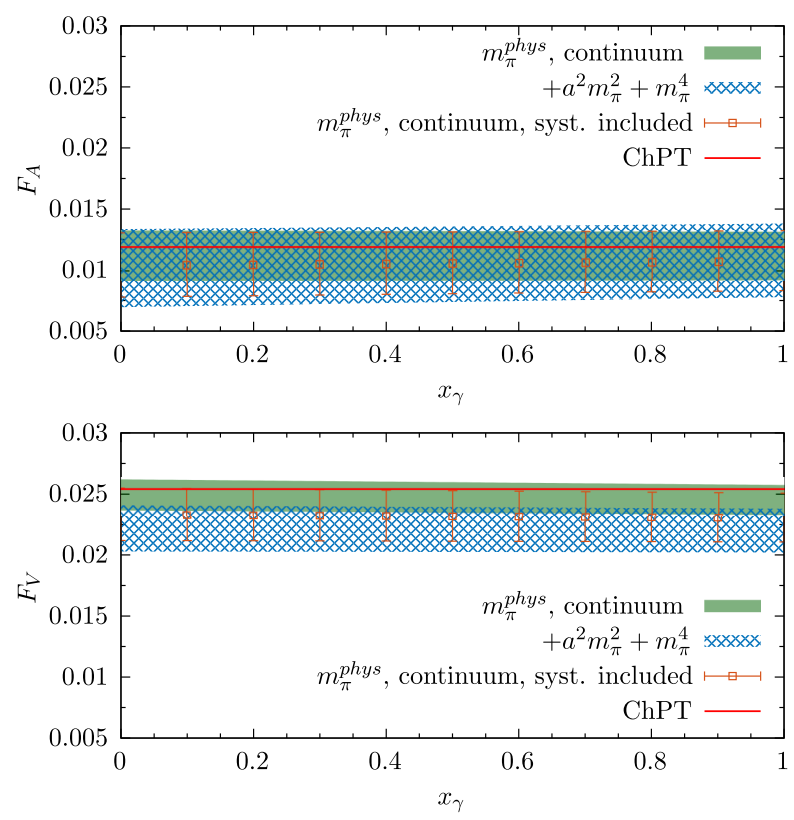

together with the corresponding statistical and systematic uncertainties. The systematic uncertainties were estimated from the differences in the results coming from different fits of higher-order terms in the meson masses, the inclusion of different possible discretization corrections
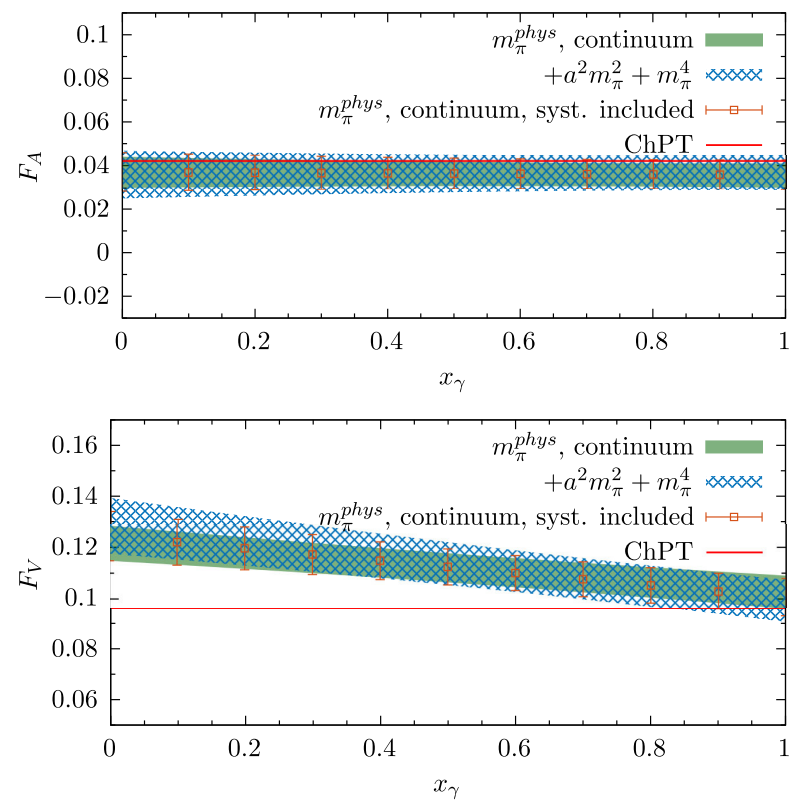

FIG. 10. Extracted values of the pion (left) and kaon (right) form factors $F_{A}\left(x_{\gamma}\right)$ (upper) and $F_{V}\left(x_{\gamma}\right)$ (lower) as a function of $x_{\gamma}$. The horizontal red lines correspond to lowest order ChPT predictions in Eq. (33). The full green bands are the results of the fits after the continuum and chiral extrapolations obtained using Eqs. (29) and (31) for the pion and kaon, respectively, and the shaded blue bands are obtained using (30) or (32). We also show the extrapolated form factors and the corresponding uncertainties (statistical and systematic) for selected values of $x_{\gamma}$. 
and the functional forms of the fits, that is, whether we use Eqs. (29) and (31) for the pion and kaon, respectively, or Eqs. (30) and (32). The results for the form factors $F_{A, V}$ at selected values of $x_{\gamma}$, the corresponding uncertainties $\Delta_{F_{A, V}}$, and their correlation matrices are given for all the mesons in Appendix D.

For heavy mesons $H$, we expect that the form factors scale as $m_{h}^{-3 / 2} \sim f_{H} / m_{H}$, where $m_{h}$ is the mass of the heavy quark contained in $H$,

$F_{A, V}\left(x_{\gamma}\right)=F_{A, V}^{0} \frac{f_{H}}{m_{H}}\left(1+O\left(\frac{\Lambda_{\mathrm{QCD}}}{m_{H}}\right)+\ldots\right)+O\left(a^{2} m_{H}^{2}\right)$,

where the constants $F_{A, V}^{0}$ are a function of the light-quark masses. Since, however, for this exploratory study, we have only two values of the heavy-quark mass, both around the charm mass, we prefer to interpolate the values of the form factors to the physical charm quark mass and then to fit the result with the simple formula

$$
\begin{aligned}
F_{A, V}\left(x_{\gamma}\right)= & d_{0}+d_{0}^{\prime} \frac{m_{\pi}^{2}}{\left(4 \pi f_{\pi}\right)^{2}}+\tilde{d}_{0} \frac{a^{2}}{r_{0}^{2}} \\
& +\left(d_{1}+d_{1}^{\prime} \frac{m_{\pi}^{2}}{\left(4 \pi f_{\pi}\right)^{2}}+\tilde{d}_{1} \frac{a^{2}}{r_{0}^{2}}\right) x_{\gamma}
\end{aligned}
$$
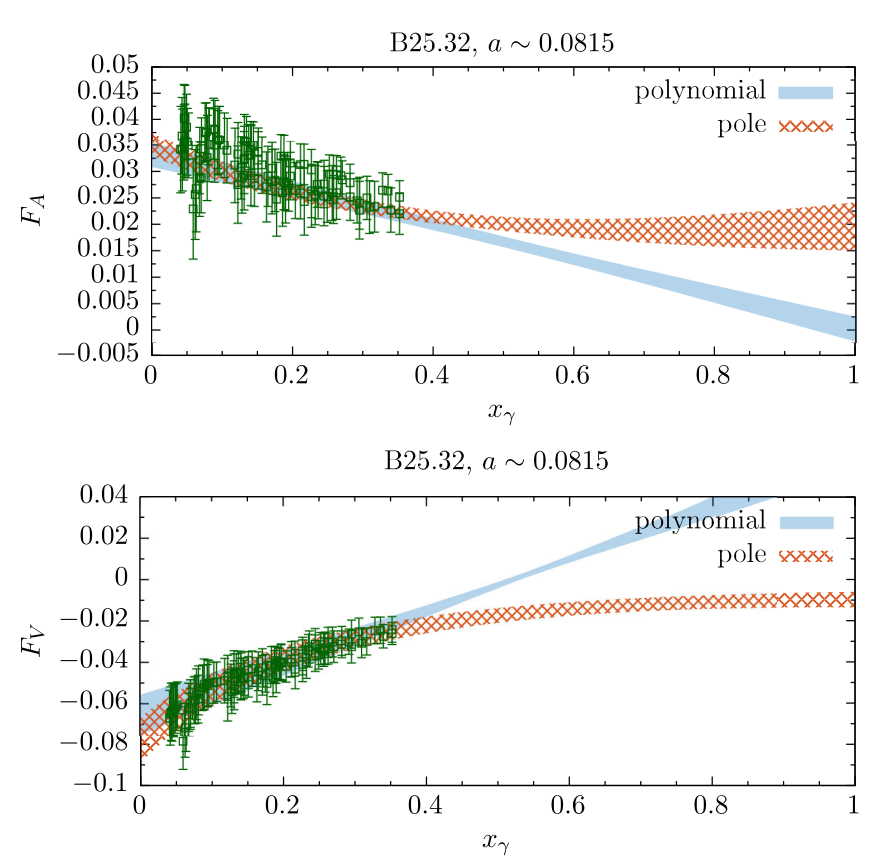

FIG. 11. The form factors $F_{A}\left(x_{\gamma}\right)$ (upper) and $F_{V}\left(x_{\gamma}\right)$ (lower) of the $D_{s}$ meson as a function of $x_{\gamma}$ at fixed lattice spacing $(a=0.0815 \mathrm{fm})$ for the ensemble B25.32 [15]. The full blue and shaded orange bands are the results of the fits with the polynomial or pole formulas given in Eqs. (35) and (36), respectively.
We have also performed fits with the polelike formula

$$
F_{A, V}\left(x_{\gamma}\right)=\frac{d_{0}+d_{0}^{\prime} \frac{m_{\pi}^{2}}{\left(4 \pi f_{\pi}\right)^{2}}}{1+\left(\Delta_{1}+\Delta_{1}^{\prime} \frac{m_{\pi}^{2}}{\left(4 \pi f_{\pi}\right)^{2}}\right) x_{\gamma}}+\tilde{d}_{0} \frac{a^{2}}{r_{0}^{2}}+\tilde{d}_{1} \frac{a^{2}}{r_{0}^{2}} x_{\gamma} .
$$

In this first study, we only have results for the $D_{(s)}$ mesons in the range $0 \leq x_{\gamma} \leq 0.4$, corresponding to $E_{\gamma} \lesssim$ $400 \mathrm{MeV}$ in the rest frame of the hadron. In Fig. 11, we give results for the form factors of the $D_{s}$ meson, $F_{A}\left(x_{\gamma}\right)$ and $F_{V}\left(x_{\gamma}\right)$, at $a=0.0815 \mathrm{fm}$. The full blue and shaded orange bands are the results of the fits with the polynomial or pole formula given in Eq. (35) and (36), respectively. Since the lattice spacing is fixed, the coefficients $\tilde{d}_{0,1}$ are not included in the fit. We see that the both the fits give a good description of our results in the region where we have data, but differ significantly for $x_{\gamma} \geq 0.4$. This means that, although both the linear and the pole fit describe accurately the form factors in the region in which we have data, it is not reliable to use these fits in the region $x_{\gamma} \geq 0.4$. In our future investigations, we plan to provide nonperturbative data for the form factors in the full kinematical range $0 \leq x_{\gamma} \leq 1-m_{\ell}^{2} / m_{D_{(s)}}^{2}$.

In Fig. 12, we present the values of the form factors $F_{A}\left(x_{\gamma}\right)$ (upper) and $F_{V}\left(x_{\gamma}\right)$ (lower) for the $D_{s}$ meson as a
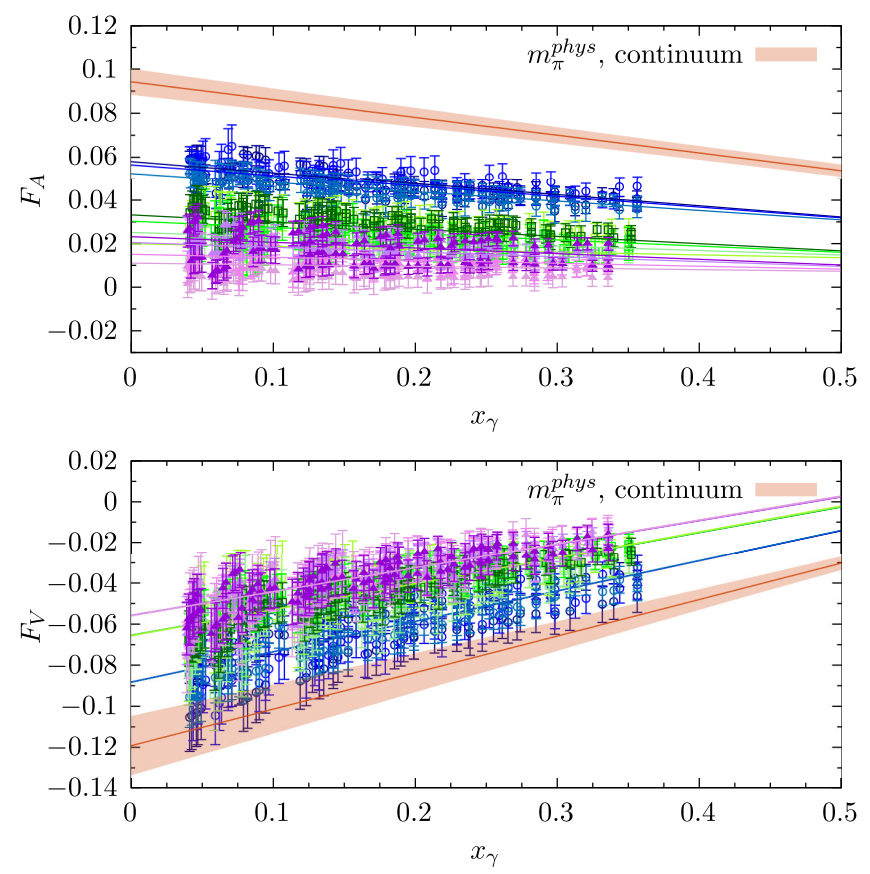

FIG. 12. The form factors $F_{A}\left(x_{\gamma}\right)$ (upper) and $F_{V}\left(x_{\gamma}\right)$ (lower) of the $D_{s}$ meson as a function of $x_{\gamma}$ at three values of the lattice spacing with separate fits to the data using Eq. (35) at each value of the lattice spacing. The orange bands with their central red lines represent the result of a single fit to all the data extrapolated to the continuum limit and to physical quark masses. 

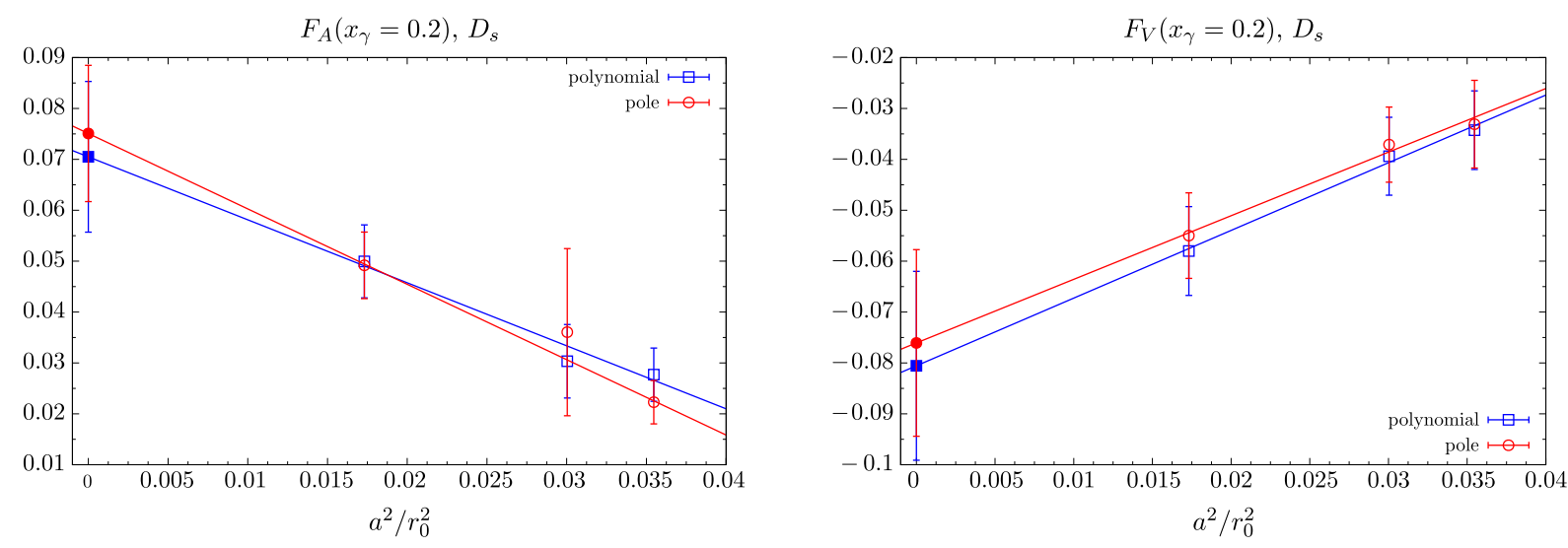

FIG. 13. The form factors $F_{A}$ (left) and $F_{V}$ (right) of the $D_{s}$ meson at $x_{\gamma}=0.2$ as functions of $a^{2}$. The polynomial and pole fits correspond to Eqs. (35) and (36), respectively.

function of $x_{\gamma}$. We show the data obtained at the three different values of the lattice spacing, together with fits using Eq. (35) at each value of the lattice spacing. The orange bands with their central red lines are the results of a single fit to all the data after extrapolation to the continuum limit and to physical quark masses. The discretization artifacts, which include ones of $O\left(m_{c}^{2} a^{2}\right)$, while approximately of the expected size, appear to be relatively large because the form factors are small. In fact, the form factors at the three lattice spacings we have at our disposal are fully consistent, within our uncertainties, with a linear behavior in $a^{2}$, as illustrated in Fig. 13 where the form factors at $x_{\gamma}=0.2$ are presented as a function of the lattice spacing. The points in the figure are obtained after extrapolation to physical quark masses either using a polynomial of pole
Ansatz corresponding to Eq. (35) or (36) at fixed lattice spacing. In this first study, with only three lattice spacings at our disposal, we are unable to include corrections of higher order in $a^{2}$ beyond those present in Eqs. (35) and (36). In Appendix D, we have estimated their effects in the uncertainties of our final results for the form factors.

We also study our physical results (i.e those obtained after the continuum and chiral extrapolations) as a function of $x_{\gamma}$ by fitting them to the following linear expressions:

$$
F_{A, V}^{P}\left(x_{\gamma}\right)=C_{A, V}^{P}+D_{A, V}^{P} x_{\gamma},
$$

where $P$ represents each of the pseudoscalar mesons, $\pi, K$, $D$, and $D_{s}$.

For the axial form factors, we find

$$
\begin{aligned}
& C_{A}^{\pi}=0.010 \pm 0.003, \quad D_{A}^{\pi}=0.0004 \pm 0.0006, \quad \rho_{C_{A}^{\pi}, D_{A}^{\pi}}=-0.419, \\
& C_{A}^{K}=0.037 \pm 0.009, \quad D_{A}^{K}=-0.001 \pm 0.007, \quad \rho_{C_{A}^{K}, D_{A}^{K}}=-0.673, \\
& C_{A}^{D}=0.109 \pm 0.009, \quad D_{A}^{D}=-0.10 \pm 0.03, \quad \rho_{C_{A}^{D}, D_{A}^{D}}=-0.557, \\
& C_{A}^{D_{s}}=0.092 \pm 0.006, \quad D_{A}^{D_{s}}=-0.07 \pm 0.01, \quad \rho_{C_{A}^{D_{s}}, D_{A}^{D_{s}}}=-0.745 \text {, }
\end{aligned}
$$

and for the vector form factors we obtain

$$
\begin{array}{cc}
C_{V}^{\pi}=0.023 \pm 0.002, & D_{V}^{\pi}=-0.0003 \pm 0.0003, \quad \rho_{C_{V}^{\pi}, D_{V}^{\pi}}=-0.570, \\
C_{V}^{K}=0.12 \pm 0.01, & D_{V}^{K}=-0.02 \pm 0.01, \quad \rho_{C_{V}^{K}, D_{V}^{K}}=-0.714, \\
C_{V}^{D}=-0.15 \pm 0.02, & D_{V}^{D}=0.12 \pm 0.04, \quad \rho_{C_{V}^{D}, D_{V}^{D}}=-0.580, \\
C_{V}^{D_{s}}=-0.12 \pm 0.02, & D_{V}^{D_{s}}=0.16 \pm 0.03, \quad \rho_{C_{V}^{D_{s}}, D_{V}^{D_{s}}}=-0.900 .
\end{array}
$$

In Eqs. (38) and (39), for each of the $C$ 's and $D$ 's, $\rho_{C, D}$ is the correlation between them, defined by

$$
\rho_{C, D}=\frac{\sum_{i}\left(C_{i}-\mu_{C}\right)\left(D_{i}-\mu_{D}\right)}{\sqrt{\sum_{i}\left(C_{i}-\mu_{C}\right)^{2}} \sqrt{\sum_{i}\left(D_{i}-\mu_{D}\right)^{2}}}, \quad \mu_{C}=\frac{1}{N} \sum_{i} C_{i}, \quad \mu_{D}=\frac{1}{N} \sum_{i} D_{i}
$$


where $C_{i}$ and $D_{i}$ are the jackknife samples and the sum runs over all the jackknifes following the procedure in Appendix A of Ref. [29]. The full correlation matrices are given in Appendix E.

For the pion and kaon, we can compare the constants $C_{A, V}^{\pi, K}$ in Eqs. (38) and (39) with the constant (i.e. $x_{\gamma^{-}}$ independent) values obtained in ChPT using Eq. (33): $F_{A}^{\pi}=0.0119, F_{V}^{\pi}=0.0254, F_{A}^{K}=0.042, F_{V}^{K}=0.096$.

The pion vector form factor $F_{V}^{\pi}$ was computed on the lattice in Ref. [30] where the process $\pi^{0} \rightarrow \gamma \gamma$ was studied. The main difference between Ref. [30] and our approach is that in that case the space momentum $\boldsymbol{p}$ of the meson and of one of the two photons, let us call it $\boldsymbol{q}$, was fixed, whereas the space momentum of the remaining photon, corresponding to our $\boldsymbol{k}$, was given by momentum conservation. In general, then, the two photons are off-massshell, and an extrapolation to the on-shell condition $q^{2}=$ $k^{2}=0$ is performed in order to obtain the form factor at the physical point. The authors give their form factor, defined as $F\left(m_{\pi}^{2}, q^{2}, k^{2}\right)=F_{V}^{\pi}\left(4 \pi^{2} f_{\pi}\right) / m_{\pi}$ at the points $F\left(m_{\pi}^{2}, 0,0\right)=$ $1.005(20)(30)$ and $F(0,0,0)=1.009(22)(29)$, whereas we have the form factor as a function of $q^{2}$, which is fixed by $x_{\gamma}$. Given the smooth dependence of the vector form factor on the momenta, we can nevertheless make a comparison of these results with ours, namely, with $\left(C_{V}^{\pi}+D_{V}^{\pi}\right)\left(4 \pi^{2} f_{\pi}\right) / m_{\pi}=0.88(8)$. The results are compatible within the errors.

In the remainder of this section, we present a brief comparison of our results with experimental data. A more detailed phenomenological analysis will be presented in a separate paper.

For the pion, the Particle Data Group (PDG) [1] quotes the following results: $F_{A}^{\pi}=0.0119$ (1) (this value comes from fixing the vector form factor at the conserved vector current prediction from $\pi^{0} \rightarrow \gamma \gamma$ decays, $\left.F_{V}^{\pi}\left(x_{\gamma}=0\right)=1 / \alpha \sqrt{2 \Gamma\left(\pi^{0} \rightarrow \gamma \gamma\right) /\left(\pi m_{\pi^{0}}\right)}=0.0259(5)\right)$, and
$F_{V}^{\pi}=0.025(2)$ in nice agreement with our results, respectively, $F_{A}^{\pi}=C_{A}^{\pi}=0.010(3)$ and $F_{V}^{\pi}=C_{V}^{\pi}=0.023(2)$. Also the slope of $F_{V}^{\pi}\left(x_{\gamma}\right)$ has been measured from the expression $F_{V}^{\pi}\left(x_{\gamma}\right)=F_{V}^{\pi}(1)\left(1+\lambda\left(1-x_{\gamma}\right)\right)$ with the result $\lambda=0.10(6)$, to be compared with our result $\lambda=-D_{V}^{\pi} /\left(C_{V}^{\pi}+D_{V}^{\pi}\right)=0.011(12)$.

For the kaon, the PDG quotes the two combinations $F_{V}^{K} \pm F_{A}^{K}$. They present separate values obtained from $K \rightarrow e$ decays, $F_{V}^{K}+F_{A}^{K}=0.133(8)$, and from $K \rightarrow \mu$ decays, $F_{V}^{K}+F_{A}^{K}=0.165(13)$. Of course, the results should be independent of whether the final-state charged lepton is an electron or muon. For this combination of form factors, our value is $F_{V}^{K}+F_{A}^{K}=0.161 \pm 0.013$ at $x_{\gamma}=0$ and $F_{A}^{K}+$ $F_{V}^{K}=0.1363 \pm 0.0096$ at $x_{\gamma}=1$. For the other combination of form factors, the PDG quotes $F_{A}^{K}-F_{V}^{K}=-0.21(6)$ obtained from $K \rightarrow \mu$ decays, which is quite different from our result $F_{A}^{K}-F_{V}^{K}=-0.087 \pm 0.013$ at $x_{\gamma}=0$ or $F_{A}^{K}-$ $F_{V}^{K}=-0.06 \pm 0.01$ at $x_{\gamma}=1$. From $K \rightarrow e$ decays, there is only the upper bound $F_{A}^{K}(0)-F_{V}^{K}(0)<0.49$.

The results in Eqs. (38) and (39) can be combined with the values of the decays constants computed in Refs. [19,31],

$$
\begin{array}{ll}
f_{\pi}=(130.41 \pm 0.20) \mathrm{MeV} & f_{K}=(155.0 \pm 1.9) \mathrm{MeV} \\
f_{D}=(207.4 \pm 3.8) \mathrm{MeV} & f_{D_{s}}=(247.2 \pm 4.1) \mathrm{MeV}
\end{array}
$$

to compute the differential or total decay rate using the expressions given in Appendix A.

For completeness, we also present the constants $\tilde{C}_{A, V}^{D_{(s)}}$ and $\tilde{D}_{A, V}^{D_{(s)}}$ which appear in the pole representation of the form factors for $D$ and $D_{s}$ mesons,

$$
F_{A, V}^{D_{(s)}}\left(x_{\gamma}\right)=\frac{\tilde{C}_{A, V}^{D_{(s)}}}{1+\tilde{D}_{A, V}^{D_{(s)} x_{\gamma}}}:
$$

\section{CONCLUSIONS}

In conclusion, we have shown that by using lattice QCD, even with moderate statistics, it is possible to predict with good precision the structure-dependent form factors $F_{A}$ and $F_{V}$ relevant for $P \rightarrow \ell \bar{\nu}_{\ell} \gamma$ decays for both light and heavy mesons and that it is also possible to extract their momentum dependence. Previous determinations of these quantities relied either on ChPT for light mesons or on the heavy-quark expansion and model-dependent assumptions for heavy mesons. Our work shows that it is possible to compute the relevant form factors from first principles. 
We found that the extraction of the axial form factor $F_{A}$ at small values of $x_{\gamma}$ is problematic because of the presence of very large discretization effects of $O\left(a^{2} /\left(r_{0}^{2} x_{\gamma}\right)\right)$ and we provided a procedure for the nonperturbative cancellation of these systematic errors. We also found that for charmed mesons the discretization effects of $O\left(a^{2} m_{H}^{2}\right)$, while of the expected order of magnitude, are large relative to the small size of the form factors. Nevertheless, the results for the form factors at the three lattice spacings are consistent, within our uncertainties, with a linear behavior in $a^{2}$. Simulations on one or more finer lattices would enable us to improve our estimates of the higher-order artifacts and hence reduce the corresponding systematic uncertainty. Such preliminary studies of charmed mesons are also essential in order to study radiative decays of $B$ mesons in the future. In this respect, the use of the ratio method may also be very useful [32].

Although the present study clearly can and will be improved by, for example, increasing the statistics, covering the full range of $x_{\gamma}$ for $D$ and $D_{s}$ mesons or simulating on a finer lattice, the results presented in this work already allow for an accurate comparison of the theoretical predictions with experimental measurements and we will discuss the phenomenological implications of our results in a forthcoming paper.

In future, we also plan to study the emission of off-shell photons $\left(k^{2} \neq 0\right)$, computing all four form factors appearing in Eq. (3), which would allow us to predict the rates for processes in which the pseudoscalar meson decays into four leptons. These processes are very interesting in the search of physics beyond the Standard Model [33-35].

\section{ACKNOWLEDGMENTS}

We gratefully acknowledge helpful discussions with M. Testa. We acknowledge PRACE for awarding us access to Marconi at CINECA, Italy under the Grant No. Pra174394. We also acknowledge use of CPU time provided by CINECA under the specific initiative INFN-LQCD123. V.L., G. M., and S.S. thank MIUR (Italy) for partial support under the contract PRIN 2015. The work of C. T. S. was partially supported by STFC (UK) Grant No. ST/P000711/1 and by an Emeritus Fellowship from the Leverhulme Trust. N. T. and R. F. acknowledge the University of Rome Tor Vergata for the support granted to the project PLNUGAMMA. The work of F. S. and S. S. was supported by the Italian Ministry of Research (MIUR) under Grant No. PRIN 20172LNEEZ. The work of F. S. was supported by INFN under GRANT73/CALAT.

\section{APPENDIX A: EXPRESSIONS FOR THE DECAY RATES IN TERMS OF $F_{V}$ AND $F_{A}$}

In this appendix, we present the explicit formulas needed to evaluate the total and differential decay rates at order $\alpha_{\mathrm{em}}$, combining the nonperturbative determination of the virtual corrections computed with the approach of Ref. [10] with the calculation of the SD form factors $F_{A}$ and $F_{V}$ determined with the method proposed in this paper. These formulas can be used to compute the double differential decay rates $d^{2} \Gamma /\left(d x_{\gamma} d x_{\ell}\right)$, the single differential decay rates, $d \Gamma / d x_{\ell}$ or $d \Gamma / d x_{\gamma}$, as well as the integrated decay rate $\Gamma\left(\Delta E_{\gamma}\right)=\int_{0}^{2 \Delta E_{\gamma} / m_{P}} d x_{\gamma}\left(d \Gamma / d x_{\gamma}\right)\left(\Delta E_{\gamma}\right.$ is the upper limit on the energy of the emitted photon in the meson rest frame).

The exchange of a virtual photon depends on the hadron structure, since all momentum modes are included, and the amplitude must therefore be computed nonperturbatively. On the other hand, the nonperturbative evaluation of the amplitude for the emission of a real photon is not strictly necessary [10]. Indeed, it is possible to compute the amplitudes for real-photon emission in perturbation theory when $x_{\gamma}$ is sufficiently small that the internal structure of the decaying meson is not resolved. The infrared divergences in the nonperturbatively computed amplitude with the exchange of a virtual photon are canceled in the decay rates by those present in the emission of a real photon, even when the latter is computed perturbatively. The reason for this cancellation is the universality of the infrared behavior of the theory (i.e. the infrared divergences do not depend on the structure of the decaying hadron). For large photon energies, for example, those present in the decays of heavy mesons, a full nonperturbative determination of the relevant amplitudes is necessary.

To calculate the partial rates for the emission of a hard real photon, it is sufficient to know the SD form factors, $F_{A}$ and $F_{V}$, and the meson's decay constant $f_{P}$. For the integrated rate $\Gamma\left(\Delta E_{\gamma}\right)$ instead, in the intermediate steps of the calculation, it is necessary to introduce an infrared regulator. To this end, in order to work with quantities that are finite when the infrared regulator is removed, it is very useful to organize the inclusive rate $\Gamma\left(\Delta E_{\gamma}\right)=\Gamma\left(P^{-} \rightarrow\right.$ $\left.\ell^{-} \bar{\nu}_{\ell}(\gamma)\right)\left.\right|_{E_{\gamma} \leq \Delta E_{\gamma}}$ as follows:

$$
\begin{aligned}
\Gamma\left(\Delta E_{\gamma}\right)= & \lim _{L \rightarrow \infty}\left[\Gamma_{0}(L)-\Gamma_{0}^{\mathrm{pt}}(L)\right] \\
& +\lim _{\mu_{\gamma} \rightarrow 0}\left[\Gamma_{0}^{\mathrm{pt}}\left(\mu_{\gamma}\right)+\Gamma_{1}^{\mathrm{pt}}\left(\Delta E_{\gamma}, \mu_{\gamma}\right)\right] \\
& +\left[\Gamma_{1}\left(\Delta E_{\gamma}\right)-\Gamma_{1}^{\mathrm{pt}}\left(\Delta E_{\gamma}\right)\right]
\end{aligned}
$$

where the subscripts 0,1 indicate the number of photons in the final state, while the superscript pt denotes the pointlike approximation of the decaying meson and $\mu_{\gamma}$ is an infrared regulator. On the right-hand side of Eq. (A1), the quantities $\Gamma_{0}(L)$ and $\Gamma_{1}\left(\Delta E_{\gamma}\right)$ are evaluated on the lattice.

The terms in the first parentheses on the right-hand side of Eq. (A1), $\Gamma_{0}(L)$ and $\Gamma_{0}^{\mathrm{pt}}(L)$, have the same infrared divergences which therefore cancel in the difference. Here we use the lattice size $L$ as the intermediate infrared regulator by working in the $\mathrm{QED}_{\mathrm{L}}$ formulation of $\mathrm{QED}$ 
in a finite volume [36], but any other consistent formulation of QED on the lattice can also be used. The difference $\left[\Gamma_{0}-\Gamma_{0}^{\mathrm{pt}}\right]$ is independent of the regulator as this is removed [11]. $\Gamma_{0}(L)$ depends on the structure of the decaying meson and is computed nonperturbatively, Refs. [11-15].

In the terms in the second parentheses on the righthand side of Eq. (A1), the decaying meson is taken to be a pointlike charged particle and both $\Gamma_{0}^{\mathrm{pt}}\left(\mu_{\gamma}\right)$ and $\Gamma_{1}^{\mathrm{pt}}\left(\Delta E_{\gamma}, \mu_{\gamma}\right)$ can be computed directly in infinite volume, in perturbation theory, using some infrared regulator, for example, a photon mass $\mu_{\gamma}=m_{\gamma}$. Each term is infrared divergent, but the sum is convergent [2] and independent of the infrared regulator. In Refs. [10,11], the explicit perturbative calculations of $\left[\Gamma_{0}^{\mathrm{pt}}\left(\mu_{\gamma}\right)+\Gamma_{1}^{\mathrm{pt}}\left(\Delta E_{\gamma}, \mu_{\gamma}\right)\right]$ and $\Gamma_{0}^{\mathrm{pt}}(L)$ have been performed with a small photon mass $\mu_{\gamma}$ or using the finite volume, respectively, as the infrared regulators.

Finally, the term on second line of the right-hand side of Eq. (A1) is infrared finite. It can be computed in the infinite-volume limit requiring only knowledge of the structure-dependent form factors, $F_{A}\left(x_{\gamma}\right)$ and $F_{V}\left(x_{\gamma}\right)$, and of the meson's decay constant $f_{P}$,

$$
\left[\Gamma_{1}\left(\Delta E_{\gamma}\right)-\Gamma_{1}^{\mathrm{pt}}\left(\Delta E_{\gamma}\right)\right]=\Gamma_{\mathrm{SD}}\left(\Delta E_{\gamma}\right)+\Gamma_{\mathrm{INT}}\left(\Delta E_{\gamma}\right),
$$

where $\Gamma_{\mathrm{SD}}$ is the structure-dependent contribution and $\Gamma_{\mathrm{INT}}$ is that from the interference between the SD and pointlike components of the amplitudes. Both $\Gamma_{\mathrm{SD}}$ and $\Gamma_{\mathrm{INT}}$ are separately infrared finite and there is no need to introduce an infrared regulator in this term.
We express the differential decay rate in terms of the following quantities:

(i) The two dimensionless kinematical variables

$$
x_{\gamma}=\frac{2 p \cdot k}{m_{P}^{2}}, \quad x_{\ell}=\frac{2 p \cdot p_{\ell}-m_{\ell}^{2}}{m_{P}^{2}},
$$

where $m_{\ell}$ the mass of the lepton $\ell, 1-x_{\gamma}+x_{\gamma} r_{\ell}^{2} /$ $\left(1-x_{\gamma}\right) \leq x_{\ell} \leq 1$, and $0 \leq x_{\gamma} \leq 1-r_{\ell}^{2}$, with $r_{\ell}=m_{\ell} / m_{P}$.

(ii) The decay constant of the meson $f_{P}$.

(iii) The two $\mathrm{SD}$ axial and vector form factors $F_{A}$ and $F_{V}$.

The differential decay rate is given by the sum of three contributions,

$\frac{d^{2} \Gamma}{d x_{\gamma} d x_{\ell}}=\frac{\alpha_{\mathrm{em}} \Gamma^{(0)}}{4 \pi}\left\{\frac{d^{2} \Gamma_{\mathrm{pt}}}{d x_{\gamma} d x_{\ell}}+\frac{d^{2} \Gamma_{\mathrm{SD}}}{d x_{\gamma} d x_{\ell}}+\frac{d^{2} \Gamma_{\mathrm{INT}}}{d x_{\gamma} d x_{\ell}}\right\}$

where $\Gamma^{(0)}$ is the leptonic decay rate in the absence of electromagnetic corrections. This is given by

$$
\Gamma^{(0)}=\frac{G_{F}^{2}\left|V_{\mathrm{CKM}}\right|^{2} f_{P}^{2}}{8 \pi} m_{P}^{3} r_{\ell}^{2}\left(1-r_{\ell}^{2}\right)^{2}
$$

where $G_{F}$ is the Fermi's constant and $V_{\mathrm{CKM}}$ the relevant CKM matrix element.

The quantities in the braces on the right-hand side of Eq. (A4) are given by

$$
\begin{aligned}
\frac{d^{2} \Gamma_{\mathrm{pt}}}{d x_{\gamma} d x_{\ell}} & =\frac{2 f_{\mathrm{pt}}\left(x_{\gamma}, x_{\ell}\right)}{\left(1-r_{\ell}^{2}\right)^{2}}, \\
\frac{d^{2} \Gamma_{\mathrm{SD}}}{d x_{\gamma} d x_{\ell}} & =\frac{m_{P}^{2}\left\{\left[F_{V}\left(x_{\gamma}\right)+F_{A}\left(x_{\gamma}\right)\right]^{2} f_{\mathrm{SD}}^{+}\left(x_{\gamma}, x_{\ell}\right)+\left[F_{V}\left(x_{\gamma}\right)-F_{A}\left(x_{\gamma}\right)\right]^{2} f_{\mathrm{SD}}^{-}\left(x_{\gamma}, x_{\ell}\right)\right\}}{2 f_{P}^{2} r_{\ell}^{2}\left(1-r_{\ell}^{2}\right)^{2}} \\
\frac{d^{2} \Gamma_{\mathrm{INT}}}{d x_{\gamma} d x_{\ell}} & =-\frac{2 m_{P}\left\{\left[F_{V}\left(x_{\gamma}\right)+F_{A}\left(x_{\gamma}\right)\right] f_{\mathrm{INT}}^{+}\left(x_{\gamma}, x_{\ell}\right)+\left[F_{V}\left(x_{\gamma}\right)-F_{A}\left(x_{\gamma}\right)\right] f_{\mathrm{INT}}^{-}\left(x_{\gamma}, x_{\ell}\right)\right\}}{f_{P}\left(1-r_{\ell}^{2}\right)^{2}}
\end{aligned}
$$

and correspond to the contribution of the pointlike approximation, to the SD contribution and to the interference between pointlike and SD terms (INT), respectively. The kinematical functions appearing in Eq. (A6) are given by

$$
\begin{aligned}
f_{\mathrm{pt}}\left(x_{\gamma}, x_{\ell}\right) & =\frac{1-x_{\ell}}{x_{\gamma}^{2}\left(x_{\gamma}+x_{\ell}-1\right)}\left[x_{\gamma}^{2}+2\left(1-x_{\gamma}\right)\left(1-r_{\ell}^{2}\right)-\frac{2 x_{\gamma} r_{\ell}^{2}\left(1-r_{\ell}^{2}\right)}{x_{\gamma}+x_{\ell}-1}\right], \\
f_{\mathrm{SD}}^{+}\left(x_{\gamma}, x_{\ell}\right) & =\left(x_{\gamma}+x_{\ell}-1\right)\left[\left(x_{\gamma}+x_{\ell}-1+r_{\ell}^{2}\right)\left(1-x_{\gamma}\right)-r_{\ell}^{2}\right], \\
f_{\mathrm{SD}}^{-}\left(x_{\gamma}, x_{\ell}\right) & =-\left(1-x_{\ell}\right)\left[\left(x_{\ell}-1+r_{\ell}^{2}\right)\left(1-x_{\gamma}\right)-r_{\ell}^{2}\right], \\
f_{\mathrm{INT}}^{+}\left(x_{\gamma}, x_{\ell}\right) & =-\frac{1-x_{\ell}}{x_{\gamma}\left(x_{\gamma}+x_{\ell}-1\right)}\left[\left(x_{\gamma}+x_{\ell}-1+r_{\ell}^{2}\right)\left(1-x_{\gamma}\right)-r_{\ell}^{2}\right], \\
f_{\mathrm{INT}}^{-}\left(x_{\gamma}, x_{\ell}\right) & =\frac{1-x_{\ell}}{x_{\gamma}\left(x_{\gamma}+x_{\ell}-1\right)}\left[x_{\gamma}^{2}+\left(x_{\gamma}+x_{\ell}-1+r_{\ell}^{2}\right)\left(1-x_{\gamma}\right)-r_{\ell}^{2}\right] .
\end{aligned}
$$


The distribution with respect to the photon's momentum is obtained after integrating over the lepton's momentum

$$
\frac{d \Gamma}{d x_{\gamma}}=\int_{x_{\ell}^{\min }\left(x_{\gamma}\right)}^{1} d x_{\ell} \frac{d^{2} \Gamma}{d x_{\gamma} d x_{\ell}} .
$$

As $x_{\gamma} \rightarrow 0$, the allowed kinematical range for $x_{\ell}$ is squeezed around its maximum, $x_{\ell}^{\min }\left(x_{\gamma}\right)=1-x_{\gamma}+x_{\gamma} r_{\ell}^{2} /$ $\left(1-x_{\gamma}\right) \leq x_{\ell} \leq 1$. Thus, with the exception of the contribution proportional to $f_{\mathrm{pt}}\left(x_{\gamma}, x_{\ell}\right) \sim 1 / x_{\gamma}^{2}$, all the other contributions vanish in the soft-photon region, which is consequently dominated by the pointlike (eikonal) result

$$
\frac{d \Gamma}{d x_{\gamma}} \sim \int_{x_{\ell}^{\min }\left(x_{\gamma}\right)}^{1} d x_{\ell} \frac{d^{2} \Gamma_{\mathrm{pt}}}{d x_{\gamma} d x_{\ell}} \sim 1 / x_{\gamma} .
$$

The $1 / x_{\gamma}$ behavior of the differential rate at small $x_{\gamma}$ leads to a logarithmic infrared divergence in the total rate. It is canceled by the infrared divergence in the $O\left(\alpha_{\mathrm{em}}\right)$ virtual corrections to the inclusive decay rate. The SD and INT contributions vanish at small $x_{\gamma}$.

Equations (A4)-(A9) allow us to compute the spectrum $d \Gamma / d x_{\gamma}$. We advocate organizing the determination of the integrated rate in terms of the three sets of parentheses on the right-hand side of Eq. (A1). The procedure to evaluate the term in the first parentheses, $\Gamma_{0}(L)-\Gamma_{0}^{\mathrm{pt}}(L)$, is explained in detail in Ref. [10], where the explicit expression for the term in the second parentheses, $\Gamma_{0}^{\mathrm{pt}}\left(\mu_{\gamma}\right)+$ $\Gamma_{1}^{\mathrm{pt}}\left(\Delta E_{\gamma}, \mu_{\gamma}\right)$, can also be found. The third term on the righthand side of Eq. (A1), $\Gamma_{1}\left(\Delta E_{\gamma}\right)-\Gamma_{1}^{\mathrm{pt}}\left(\Delta E_{\gamma}\right)=\Gamma_{\mathrm{SD}}+\Gamma_{\mathrm{INT}}$, is the subject of this paper. As explained above, both $\Gamma_{\mathrm{SD}}$ and $\Gamma_{\mathrm{INT}}$ are infrared finite and are obtained by integrating the differential rates over the physical range of $x_{\gamma}$,

$$
\begin{aligned}
\Gamma_{\mathrm{SD}}\left(\Delta E_{\gamma}\right) & =\int_{0}^{2 \Delta E_{\gamma} / m_{P}} d x_{\gamma} \frac{d \Gamma_{\mathrm{SD}}}{d x_{\gamma}}, \\
\Gamma_{\mathrm{INT}}\left(\Delta E_{\gamma}\right) & =\int_{0}^{2 \Delta E_{\gamma} / m_{P}} d x_{\gamma} \frac{d \Gamma_{\mathrm{INT}}}{d x_{\gamma}} .
\end{aligned}
$$

\section{APPENDIX B: CALCULATING MATRIX ELEMENTS FROM FINITE EUCLIDEAN LATTICES}

In this appendix, we derive some useful formulas for the extraction of the two relevant form factors, $F_{A, V}$, from the Euclidean correlation functions expressed in terms of lattice operators on a lattice with finite-time extent $T$.

In order to construct the finite $T$ equivalent of $C_{W}^{\alpha r}(t ; \boldsymbol{k}, \boldsymbol{p})$ in Eq. (14), that we will denote as $\mathcal{C}_{W}^{\alpha r}(t, T / 2 ; \boldsymbol{k}, \boldsymbol{p})$, it is convenient to define the following hadronic correlation function at fixed $t$ and $t_{y}$ :

$$
\begin{aligned}
M_{W}^{\alpha r}\left(t_{y}, t ; \boldsymbol{k}, \boldsymbol{p}\right)= & \sum_{i=1,2,3} \epsilon_{i}^{r}(\boldsymbol{k}) \sum_{\boldsymbol{y}} \sum_{\boldsymbol{x}} e^{-i \boldsymbol{k} \cdot \boldsymbol{y}+\hat{\boldsymbol{i}} / 2)+i \boldsymbol{p} \cdot \boldsymbol{x}} \\
& \times \mathrm{T}\left\langle j_{W}^{\alpha}(t) j_{\mathrm{em}}^{i}\left(t_{y}, \boldsymbol{y}\right) P(0, \boldsymbol{x})\right\rangle_{L T},
\end{aligned}
$$

where $\langle\ldots\rangle_{L T}$ denotes the average over the gauge field configurations at finite $L$ and $T$ and we introduced suitable independent vectors $\boldsymbol{\epsilon}^{r}(\boldsymbol{k}), r=1,2$, corresponding to the physical polarizations of the emitted photon. A possible simple choice, and one in which the unphysical polarizations vanish explicitly, is given by

$$
\begin{aligned}
& \epsilon_{\mu}^{1}(\boldsymbol{k}) \equiv\left(0, \frac{-k_{1} k_{3}}{|\boldsymbol{k}| \sqrt{k_{1}^{2}+k_{2}^{2}}}, \frac{-k_{2} k_{3}}{|\boldsymbol{k}| \sqrt{k_{1}^{2}+k_{2}^{2}}}, \frac{\sqrt{k_{1}^{2}+k_{2}^{2}}}{|\boldsymbol{k}|}\right), \\
& \epsilon_{\mu}^{2}(\boldsymbol{k}) \equiv\left(0, \frac{k_{2}}{\sqrt{k_{1}^{2}+k_{2}^{2}}},-\frac{k_{1}}{\sqrt{k_{1}^{2}+k_{2}^{2}}}, 0\right) .
\end{aligned}
$$

The polarization vectors satisfy

$$
\sum_{i=1}^{3} \epsilon_{i}^{r}(\boldsymbol{k}) k_{i}=0, \quad \sum_{i=1}^{3} \epsilon_{i}^{r}(\boldsymbol{k}) \epsilon_{i}^{s}(\boldsymbol{k})=\delta_{r s} .
$$

Since in our simulations we always use $\boldsymbol{k}=(0,0,|\boldsymbol{k}|)$, the polarization vectors reduce to

$$
\epsilon_{\mu}^{1}=\left(0,-\frac{1}{\sqrt{2}},-\frac{1}{\sqrt{2}}, 0\right) \quad \epsilon_{\mu}^{2}=\left(0, \frac{1}{\sqrt{2}},-\frac{1}{\sqrt{2}}, 0\right) .
$$

The "topology" of the correlation function in Eq. (B1) is explained in Fig. 2.

(i) The incoming meson is interpolated at fixed spatial momentum $\boldsymbol{p}$ by the pseudoscalar operator $P$ placed at time $t=0$,

$$
P(0)=\sum_{x} e^{i p \cdot x} P(0, x) .
$$

(ii) The hadronic weak current $j_{W}^{\alpha}(t)$ is placed at the generic time $t$. We used a local discretization of the weak current that, in the twisted-mass discretization of the fermionic action used in this work [37], is explicitly given by

$$
\begin{aligned}
j_{W}^{\alpha}(t) & =j_{V}^{\alpha}(t)-j_{A}^{\alpha}(t), \quad j_{V}^{\alpha}(t)=Z_{A} \bar{\psi}_{U}(t) \gamma^{\alpha} \psi_{D}(t), \\
j_{A}^{\alpha}(t) & =Z_{V} \bar{\psi}_{U}(t) \gamma^{\alpha} \gamma_{5} \psi_{D}(t),
\end{aligned}
$$

where $j_{V}^{\alpha}(t)$ and $j_{A}^{\alpha}(t)$ are the vector and axial components that include the corresponding renormalization factors. Note that the renormalization factors to be used in twisted mass at maximal twist are chirally rotated with respect to the ones of standard Wilson fermions [38]. In Eq. (B6), $\psi_{U}$ indicates the field of an up-type quark that, for the mesons considered in this study, can be either an up 
or a charm. Similarly, $\psi_{D}$ can be either a down or a strange quark field. The actions of the up-type and down-type quark fields have been discretized with opposite values of the chirally rotated Wilson term in order to numerically suppress $\mathrm{O}\left(a^{2}\right)$ lattice artifacts in the meson masses $[38,39]$.

(iii) The electromagnetic current $j_{\mathrm{em}}^{\mu}\left(t_{y}, \boldsymbol{y}\right)$, carrying a three-momentum $\boldsymbol{k}$ is inserted at $y=\left(t_{y}, \boldsymbol{y}\right)$. This current is defined by

$$
j_{\mathrm{em}}^{\mu}\left(t_{y}, \boldsymbol{y}\right)=\sum_{f} q_{f} j_{f}^{\mu}\left(t_{y}, \boldsymbol{y}\right),
$$

where $f$ is the flavor index, $q_{f}$ is equal to $2 / 3$ for uptype quarks and to $-1 / 3$ for down-type quarks, and

$$
\begin{aligned}
j_{f}^{\mu}(x)= & -\left\{\bar{\psi}_{f}(x) \frac{ \pm i \gamma_{5}-\gamma^{\mu}}{2} U^{\mu}(x) \psi_{f}(x+\hat{\mu})\right. \\
& \left.-\bar{\psi}_{f}(x+\hat{\mu}) \frac{ \pm i \gamma_{5}+\gamma^{\mu}}{2} U^{\mu}(x)^{\dagger} \psi_{f}(x)\right\} .
\end{aligned}
$$

In Eq. (B8), $U_{\mu}(x)$ are the QCD link variables and the signs \pm are induced by the choice made in the case of the flavor $f$ for the sign of the chirally rotated Wilson term [17].
We have used $e^{-i \boldsymbol{k} \cdot(\boldsymbol{y}+\hat{\boldsymbol{i}} / 2)}$, rather than the simpler, standard exponent $e^{-i k \cdot y}$, for the Fourier transform of the current appearing in Eq. (B1),

$j^{r}\left(t_{y}, \boldsymbol{k}\right)=\sum_{i=1}^{3} \epsilon_{i}^{r}(k) \sum_{\boldsymbol{y}} e^{-i \boldsymbol{k} \cdot(\boldsymbol{y}+\hat{\boldsymbol{i}} / 2)} j_{\mathrm{em}}^{i}\left(t_{y}, \boldsymbol{y}\right)$.

Our choice of the exponent, which is equivalent to standard one in the continuum limit, is more convenient for the discussion of the lattice WIs since we have used the point-split exactly conserved electromagnetic current in our simulations.

(iv) A technical subtlety needs to be stressed here. As discussed in the main text, in order to choose arbitrary (nondiscretized) values of the spatial momenta for the meson and for the photon, we have introduced a "flavored" extension of the electromagnetic current (see the explanation in the caption of Fig. 3). In practice, in order to have two quarks $\left(\psi_{0}\right.$ and $\psi_{t}$, where 0 and $t$ are labels for the quark fields) having the same mass, the same electric charge, the same sign of the chirally rotated Wilson term but different boundary conditions [26], the expression to be used in the numerical calculation is

$$
e^{-i \boldsymbol{k} \cdot(\boldsymbol{x}+\hat{\boldsymbol{i}} / 2)} j_{f}^{i}(x)=-\left\{\bar{\chi}_{t}(x) \frac{ \pm i \gamma_{5}-\gamma^{i}}{2} e^{i \frac{i \pi\left(\theta_{t}^{i}+\theta_{0}^{i}\right)}{L}} U^{i}(x) \chi_{0}(x+\hat{i})-\bar{\chi}_{t}(x+\hat{i}) \frac{ \pm i \gamma_{5}+\gamma^{i}}{2} e^{-\frac{i \pi\left(\theta_{t}^{i}+\theta_{0}^{i}\right)}{L}} U^{i}(x)^{\dagger} \chi_{0}(x)\right\},
$$

where we have used the fact that [see Eq. (16)]

$$
\boldsymbol{k}=\frac{2 \pi\left(\boldsymbol{\theta}_{0}-\boldsymbol{\theta}_{t}\right)}{L}, \quad \psi_{\{0, t\}}(x+\hat{i} L)=e^{2 \pi \hat{i} \cdot \theta_{\{0, t\}}} \psi_{\{0, t\}}(x),
$$

and we have defined, as usually done in implementing twisted boundary conditions [25], the periodic fields

$$
\chi_{\{0, t\}}(x)=e^{-\frac{2 \pi i x \cdot x_{\{0, t\}}}{L}} \psi_{\{0, t\}}(x) .
$$

In all the formulas that will follow, the range of the time parameters is extended over the full lattice extension, $0 \leq t<T$ and $0 \leq t_{y}<T$.

We are now ready to define the finite- $T$ correlation function

$$
\begin{aligned}
\mathcal{C}_{W}^{\alpha r}(t, T / 2 ; \boldsymbol{k}, \boldsymbol{p})= & -i \theta(T / 2-t) \sum_{t_{y}=0}^{T}\left(\theta\left(T / 2-t_{y}\right) e^{E_{\gamma} t_{y}}+\theta\left(t_{y}-T / 2\right) e^{-E_{\gamma}\left(T-t_{y}\right)}\right) M_{W}^{\alpha r}\left(t_{y}, t ; \boldsymbol{k}, \boldsymbol{p}\right) \\
& -i \theta(t-T / 2) \sum_{t_{y}=0}^{T}\left(\theta\left(T / 2-t_{y}\right) e^{-E_{\gamma} t_{y}}+\theta\left(t_{y}-T / 2\right) e^{-E_{\gamma}\left(t_{y}-T\right)}\right) M_{W}^{\alpha r}\left(t_{y}, t ; \boldsymbol{k}, \boldsymbol{p}\right) .
\end{aligned}
$$


In the continuum and large- $T$ limits, one can readily show that for $0 \ll t \ll T / 2$,

$$
\mathcal{C}_{W}^{\alpha r}(t, T / 2 ; \boldsymbol{k}, \boldsymbol{p}) \rightarrow C_{W}^{\alpha r}(t ; \boldsymbol{k}, \boldsymbol{p})=H_{W}^{\alpha r}(k, \boldsymbol{p}) \frac{e^{-t\left(E-E_{\gamma}\right)}\langle P|P| 0\rangle}{2 E}+\cdots,
$$

where $C_{W}^{\alpha r}(t ; \boldsymbol{k}, \boldsymbol{p})$ is the correlation function introduced in the main text defined in Eq. (14), $H_{W}^{\alpha r}(k, \boldsymbol{p})$ is the physical matrix element defined in Eq. (1) and the ellipsis represent subleading exponentials.

For negative time $t$ on the other hand, that is, for time separations such that $T / 2 \ll t \ll T$, in the continuum and large- $T$ limits, we have

$$
\mathcal{C}_{W}^{\alpha r}(t, T / 2 ; \boldsymbol{k}, \boldsymbol{p}) \rightarrow\left[H_{W}^{\alpha r}(k, \boldsymbol{p})\right]^{\dagger} \frac{e^{-(T-t)\left(E-E_{\gamma}\right)}\langle 0|P| P\rangle}{2 E}+\cdots,
$$

with the ellipsis again representing the subleading exponentials.

It is useful to note that, in order to separate the axial and vector form factors, it is enough to compute separately the correlation functions corresponding to the vector, $\mathcal{C}_{V}^{\alpha r}(t, T / 2 ; \boldsymbol{k}, \boldsymbol{p})$, and the axial, $\mathcal{C}_{A}^{\alpha r}(t, T / 2 ; \boldsymbol{k}, \boldsymbol{p})$, components of the weak current. Moreover, from the properties

$$
\left[H_{A}^{\alpha r}(k, \boldsymbol{p})\right]^{\dagger}=H_{A}^{\alpha r}(k, \boldsymbol{p}), \quad\left[H_{V}^{\alpha r}(k, \boldsymbol{p})\right]^{\dagger}=-H_{V}^{\alpha r}(k, \boldsymbol{p}),
$$

we deduce the following properties of the corresponding correlation functions under time reversal:

$$
\mathcal{C}_{A}^{\alpha r}(T-t, T / 2 ; \boldsymbol{k}, \boldsymbol{p})=\mathcal{C}_{A}^{\alpha r}(t, T / 2 ; \boldsymbol{k}, \boldsymbol{p}), \quad \mathcal{C}_{V}^{\alpha r}(T-t, T / 2 ; \boldsymbol{k}, \boldsymbol{p})=-\mathcal{C}_{V}^{\alpha r}(t, T / 2 ; \boldsymbol{k}, \boldsymbol{p}) .
$$

Using these relations, the quantities

$$
H_{A}^{i r}(k, \boldsymbol{p})=\epsilon_{i}^{r} p \cdot k\left[\frac{F_{A}(p \cdot k)}{m_{P}}+\frac{f_{P}}{p \cdot k}\right], \quad H_{V}^{i r}(k, \boldsymbol{p})=i\left(E_{\gamma} \boldsymbol{\epsilon}^{r} \wedge \boldsymbol{p}-E \boldsymbol{\epsilon}^{r} \wedge \boldsymbol{k}\right)^{i} \frac{F_{V}(p \cdot k)}{m_{P}}
$$

were extracted from the ratios of the correlation functions averaged over the two temporal halves of the lattice

$$
R_{A, V}^{i r}(t, T / 2 ; \boldsymbol{k}, \boldsymbol{p})=\frac{2 E \mathcal{C}_{A, V}^{\alpha r}(t, T / 2 ; \boldsymbol{k}, \boldsymbol{p})}{e^{-t\left(E-E_{\gamma}\right)}\langle P|P| 0\rangle}=H_{A, V}^{i r}(k, \boldsymbol{p})+\ldots
$$

In all the formulas of this appendix, we have used continuum notation for the four vectors but the momentum and energy carried by the current (including the associated projectors) have to be read by performing the following substitutions:

$$
k^{i} \rightarrow \hat{k}^{i}=\frac{2}{a} \sin \left(\frac{a k^{i}}{2}\right), \quad|\boldsymbol{k}| \rightarrow|\hat{\boldsymbol{k}}|=\sqrt{\hat{\boldsymbol{k}}_{1}^{2}+\hat{\boldsymbol{k}}_{2}^{2}+\hat{\boldsymbol{k}}_{3}^{2}}, \quad E_{\gamma}=\frac{2}{a} \sinh ^{-1}\left(\frac{a|\hat{\boldsymbol{k}}|}{2}\right) .
$$

In the lattice regularization that we are using (i.e. Wilson quarks at maximal twist), Eqs. (B16) and (B17) hold for given values of the indices $\alpha$ and $r \in\{1,2\}$ only up to $\mathrm{O}\left(a^{2 n+1}\right)$ lattice artifacts (for integer $n$ ). One can show however, that, as a consequence of exact lattice symmetries (see e.g. Refs. [38,39]) and the choice of momenta and polarization vectors given in Eqs. (16) and (18), these $\mathrm{O}\left(a^{2 n+1}\right)$ cutoff effects cancel if one evaluates appropriate combinations of the relevant correlation functions, namely,

$$
\frac{1}{4} \sum_{r=1,2} \sum_{j=1,2} C_{A}^{j r}(t, T / 2 ; \mathbf{k}, \mathbf{p}) / \epsilon_{j}^{r}
$$

and

$$
\begin{aligned}
& \frac{1}{4} \sum_{r=1,2} \sum_{j=1,2} C_{V}^{j r}(t, T / 2 ; \mathbf{k}, \mathbf{p}) / F_{r, j}\left(E_{\gamma}, E\right), \\
& F_{r, j}\left(E_{\gamma}, E\right)=i\left(E_{\gamma} \epsilon^{r} \wedge \mathbf{p}-E \epsilon^{r} \wedge \mathbf{k}\right)_{j},
\end{aligned}
$$

which are precisely those that occur in Eqs. (26) and (28) of the main text. For the terms in Eqs. (B21) and (B22), the time-reflection properties of Eq. (B17) hold and the derived matrix elements, in addition to satisfying the Hermiticity properties of Eq. (B16), allow for the extraction of the form factors $F_{A}$ and $F_{V}$ with no $\mathrm{O}\left(a^{2 n+1}\right)$ lattice artifacts. Our analysis of lattice correlators leading to the results in this paper has been based on data obtained from automatically $\mathrm{O}(a)$ improved combinations of the form (B21) and (B22). 


\section{APPENDIX C: EXPLOITING THE ELECTROMAGNETIC WARD IDENTITY TO RELATE THE MATRIX ELEMENT $H_{A}^{\alpha r}(k, p)$ TO THE DECAY CONSTANT $f_{P}$}

In this appendix, we study the WI that relates the axial correlation function $C_{A}^{\alpha r}(t ; \boldsymbol{k}, \boldsymbol{p})$ to the axial-pseudoscalar correlation function and, consequently, the matrix element $H_{A}^{\alpha r}(k, \boldsymbol{p})$ to the decay constant of the meson $f_{P}$. As discussed in the main text, a careful analysis of the cutoff effects reveals that the WI does not exclude the possibility of different $O\left(a^{2}\right)$ artifacts appearing in the decay constant extracted from the three-point function and that from the two-point function.

We start with a remark about the matrix element of the axial current, determined at a finite lattice spacing $a$ and using a particular lattice discretization, which we write in the form

$$
\left\langle 0\left|j_{A}^{\alpha}(0)\right| P(\boldsymbol{p})\right\rangle=f_{P}^{L} p_{L}^{\alpha},
$$

where the combination $f_{P}^{L} p_{L}^{\alpha}$ is a vector under the orthogonal group $H(4){ }^{2}$ At finite $a$, the definition of the lattice decay constant $f_{P}^{L}$ depends on the definition that we assume for the lattice momentum $p_{L}^{\alpha}$; for example, we may choose $p_{L}^{\alpha}=p^{\alpha}$ or $p_{L}^{\alpha}=2 / a \sin \left[a p^{\alpha} / 2\right]$, where $p^{\alpha}$ is the continuum value of the momentum. In particular, we define $\hat{f}_{P}$ by $\left\langle 0\left|j_{A}^{\alpha}(0)\right| P(\boldsymbol{p})\right\rangle=\hat{f}_{P}(\boldsymbol{p}) p^{\alpha}$. Note that $\hat{f}_{P}=f_{P}+O\left(a^{2}\right)$, where $f_{P}$ is the continuum value of the decay constant.

Consider the following correlation function which is relevant to our study:

$$
\int d^{4} y d^{3} \boldsymbol{x} e^{-i k \cdot y-i p \cdot x}\left\langle 0\left|\mathrm{~T}\left[j_{A}^{\alpha}(0) j_{\mathrm{em}}^{\mu}(y) P(-t,-\boldsymbol{x})\right]\right| 0\right\rangle .
$$

With Wilson-like Fermions, such as those used in our study, at fixed lattice spacing (for simplicity in the $T \rightarrow \infty$ limit), the electromagnetic WI implies that [40]

$$
\begin{array}{r}
\frac{1}{a} \sum_{\mu=0}^{3} \int d^{4} y d^{3} x e^{-i k \cdot y-i p \cdot x}\left\langle 0\left|\mathrm{~T}\left[j_{A}^{\alpha}(0)\left\{j_{\mathrm{em}}^{\mu}(y)-j_{\mathrm{em}}^{\mu}(y-\hat{\mu})\right\} P(-x)\right]\right| 0\right\rangle \\
=-\int d^{4} y d^{3} x e^{-i k \cdot y-i p \cdot x}\left\{\delta^{4}(y)-\delta^{4}(y+x)\right\}\left\langle 0\left|\mathrm{~T}\left[j_{A}^{\alpha}(0) P(-x)\right]\right| 0\right\rangle,
\end{array}
$$

where integrals over the spatial coordinates have to be read as lattice sums and, in the case of a real photon, $k^{0}=i E_{\gamma}(\boldsymbol{k})=i|\boldsymbol{k}|$.

The WI can be rewritten in the form

$$
\sum_{i=1}^{3} \frac{2 \sin \left(a k_{i} / 2\right)}{a} C_{A}^{\alpha i}(t ; \boldsymbol{k}, \boldsymbol{p})=C_{A}^{\alpha}(t ; \boldsymbol{p})-C_{A}^{\alpha}(t ; \boldsymbol{k}, \boldsymbol{p})
$$

where we have defined [note the shift in the exponent with respect to Eq. (C3)]

$$
C_{A}^{\alpha \mu}(t ; \boldsymbol{k}, \boldsymbol{p})=-i \int d^{4} y d^{3} x e^{-i k \cdot(y+\hat{\mu} / 2)-i p \cdot x}\left\langle 0\left|\mathrm{~T}\left[j_{A}^{\alpha}(0) j_{\mathrm{em}}^{\mu}(y) P(-t,-\boldsymbol{x})\right]\right| 0\right\rangle
$$

and

$$
\begin{aligned}
C_{A}^{\alpha}(t ; \boldsymbol{p}) & =\int d^{4} y d^{3} x e^{-i k \cdot y-i \boldsymbol{p} \cdot \boldsymbol{x}} \delta^{4}(y)\left\langle 0\left|\mathrm{~T}\left[j_{A}^{\alpha}(0) P(-t,-\boldsymbol{x})\right]\right| 0\right\rangle \\
& =\int d^{3} x e^{-i \boldsymbol{p} \cdot \boldsymbol{x}}\left\langle 0\left|\mathrm{~T}\left[j_{A}^{\alpha}(0) P(-t,-\boldsymbol{x})\right]\right| 0\right\rangle \\
C_{A}^{\alpha}\left(t ; E_{\gamma}, \boldsymbol{p}-\boldsymbol{k}\right) & =\int d^{4} y d^{3} x e^{-i k \cdot y-i \boldsymbol{p} \cdot \boldsymbol{x}} \delta^{4}(y+x)\left\langle 0\left|\mathrm{~T}\left[j_{A}^{\alpha}(0) P(-t,-\boldsymbol{x})\right]\right| 0\right\rangle \\
& =\int d^{3} x e^{E_{\gamma} t-i(\boldsymbol{p}-\boldsymbol{k}) \cdot \boldsymbol{x}}\left\langle 0\left|\mathrm{~T}\left[j_{A}^{\alpha}(0) P(-t,-\boldsymbol{x})\right]\right| 0\right\rangle .
\end{aligned}
$$

\footnotetext{
${ }^{2}$ In this appendix, as in Sec. IV, the label $L$ stands for lattice, as the discussion concerns the Ward identity in a discrete space-time. It should not be confused here with the spatial extent of the lattice.
} 
We can derive the Ward identity for the matrix element itself by going onto the mass shell of the pseudoscalar meson, which in the Euclidean corresponds to selecting the energy of the external hadronic state to be $E_{P}$ as $|-t|$ becomes very large.
Consider first the case with $\boldsymbol{k} \neq 0$. In this case, the second term on the right-hand side of Eq. (C4) does not contribute because it corresponds to a different energy. Thus, in this case, we have the following identity which is true at all orders in $a$ :

$$
\begin{aligned}
\frac{2 \sin \left(k_{\mu} a / 2\right)}{a} H_{L}^{\alpha \mu}(k, \boldsymbol{p}) & =-i \frac{2 \sin \left(k_{\mu} a / 2\right)}{a} \int d^{4} y e^{-i k \cdot(y+\hat{\mu} / 2)}\left[\langle 0| \mathrm{T}\left[j_{A}^{\alpha}(0) j_{\mathrm{em}}^{\mu}(y)\right)\right]|P(\boldsymbol{p})\rangle \\
& =\left\langle 0\left|j_{A}^{\alpha}(0)\right| P(\boldsymbol{p})\right\rangle .
\end{aligned}
$$

Note that to arrive at this identity we do not need to specify the choice of $f_{P}^{L}$. As $a \rightarrow 0$, the discretized derivative in Fourier space $2 / a \sin \left(a k_{\mu} / 2\right) \rightarrow k_{\mu}$ and we recover the continuum WI in Eq. (4). We can now proceed in analogy to the continuum and separate $H_{L}^{\alpha \mu}(k, \boldsymbol{p})$ into a pointlike and a structure-dependent tensor, $H_{L}^{\alpha \mu}(k, \boldsymbol{p})=H_{L-\mathrm{pt}}^{\alpha \mu}(k, \boldsymbol{p})+H_{L-\mathrm{SD}}^{\alpha \mu}(k, \boldsymbol{p})$ such that

$$
\frac{2 \sin \left(a k_{\mu} / 2\right)}{a} H_{L-\mathrm{SD}}^{\alpha \mu}(k, \boldsymbol{p})=0
$$

at fixed $a$. Even in the continuum, the separation of $H^{\alpha \mu}$ into a pointlike and a structure-dependent component has an ambiguity in the terms, starting at $O\left(k^{2}\right)$, which are not constrained by the Ward identity or the equations of motion. Moreover, there are an infinite number of possible point-like lattice-regularized versions of $H_{L-\mathrm{pt}}^{\alpha \mu}(k, \boldsymbol{p})$ which tend to the chosen continuum one as $a \rightarrow 0$. We choose to define $H_{L-\mathrm{pt}}^{\alpha \mu}$ by

$$
H_{L-\mathrm{pt}}^{\alpha \mu}(k, \boldsymbol{p})=f_{P}^{L}\left(A(k, \boldsymbol{p}) \delta^{\alpha \mu}+\frac{T^{\alpha \mu}(k, \boldsymbol{p})}{\Delta}\right),
$$

where $\Delta^{-1}$ is some version of a lattice boson propagator, for example,

$$
\Delta^{-1}=\frac{1}{4 / a^{2} \sum_{\rho} \sin ^{2}\left[(p-k)_{\rho} a / 2\right]+m_{P}^{2}} \rightarrow \frac{1}{-2 p \cdot k+k^{2}}+O\left(a^{2}\right)
$$

as $a \rightarrow 0, A(k, \boldsymbol{p})=1+O\left(a^{2}\right)$ and $T^{\alpha \mu}(k, \boldsymbol{p})=(2 p-k)^{\mu}(p-k)^{\alpha}+O\left(a^{2}\right)$ are functions of the momenta which depend on the lattice regularization and $f_{P}^{L}$ is the meson decay constant extracted from the matrix element in Eq. (C1). We therefore have

$$
\lim _{a \rightarrow 0} H_{L-\mathrm{pt}}^{\alpha \mu}(k, \boldsymbol{p})=H_{\mathrm{pt}}^{\alpha \mu}(k, \boldsymbol{p}) .
$$

At fixed lattice spacing, the only condition that must be satisfied is that in applying the WI,

$$
\begin{aligned}
\frac{2 \sin \left(a k_{\mu} / 2\right)}{a} H_{L-\mathrm{pt}}^{\alpha \mu}(k, \boldsymbol{p}) & =f_{P}^{L}\left(\frac{2 \sin \left(k^{\alpha} a / 2\right)}{a} A(k, \boldsymbol{p})+\frac{2 \sin \left(k_{\mu} a / 2\right)}{a} \frac{T^{\alpha \mu}(k, \boldsymbol{p})}{\Delta}\right) \\
& =f_{P}^{L} p_{L}^{\alpha}=f_{P} p^{\alpha}+O\left(a^{2}\right),
\end{aligned}
$$

the denominator $\Delta$ of Eq. (C9) disappears. The WI guarantees that the right-hand side of Eq. (C12) is the matrix element $\left\langle 0\left|j_{A}^{\alpha}(0)\right| P(\boldsymbol{p})\right\rangle$ including all orders in $a$.

By iterating order by order in $a$, we may find solutions of the form

$$
\begin{aligned}
A(k, \boldsymbol{p}) & =1+a^{2} \tilde{A}\left(p^{2}\right)+O\left(a^{4}\right) \\
T^{\alpha \mu}(k, \boldsymbol{p}) & =(2 p-k)^{\mu}(p-k)^{\alpha}+a^{2} \tilde{T}^{\alpha \mu}(k, \boldsymbol{p})+O\left(a^{4}\right)
\end{aligned}
$$

that satisfy the WI, where the coefficients of the expansion are not unique. The only relevant term for the extraction of the form factor $F_{A}$, however, is the coefficient $A(k, \boldsymbol{p})$ (since $\epsilon_{\mu}^{r}(\boldsymbol{k}) T^{\alpha \mu}(k, \boldsymbol{p})=0$ ), which may differ from one by terms of $O\left(a^{2}\right)$, thus giving an effective decay constant which is different from the one naively expected from the WI. The absence of lattice artifacts of $O\left(a^{2 n+1}\right)$ is a consequence of our use of the combinations of lattice correlations functions in Eqs. (B21) and (B22) and the resulting $H_{L}^{\alpha \mu}$ matrix elements [see Eq. (C9)]. Alternatively, 
one might work with lattice formulations which preserve chiral symmetry, such as those based on overlap or domain wall fermions. For $\mathrm{O}(a)$ improved Wilson fermion lattice actions, instead, corrections of $\mathrm{O}\left(a^{3}\right)$ will in general occur.

From the above discussion, we conclude that the lattice $H_{A}^{j r}(k, \boldsymbol{p})$ has the form

$$
\begin{aligned}
H_{A}^{j r}(k, \boldsymbol{p})= & \epsilon_{j}^{r} \frac{m_{P}}{2}\left[x_{\gamma}\left(F_{A}\left(x_{\gamma}\right)+a^{2} \Delta F_{A}\left(x_{\gamma}\right)\right)\right. \\
& \left.+\frac{2}{m_{P}}\left(f_{P}+a^{2} \Delta f_{P}\right)\right]+\cdots,
\end{aligned}
$$

where the dots represent higher-order discretization corrections.

In order to implement the strategy described in Eq. (27), we need to perform a direct calculation of $H_{A}^{j r}(0, \boldsymbol{p})$ and hence to study the $k \rightarrow 0$ limit of the WI. The problem is nontrivial because from the spectral analysis of $C_{A}^{\alpha \mu}(t ; \boldsymbol{k}, \boldsymbol{p})$ it follows that

$$
C_{A}^{\alpha \mu}(t ; \boldsymbol{k}, \boldsymbol{p})=c_{1}^{\alpha \mu} e^{-t E_{P}(\boldsymbol{p})}+c_{2}^{\alpha \mu} e^{-t\left\{E_{P}(\boldsymbol{p}-\boldsymbol{k})+E_{\gamma}(\boldsymbol{k})\right\}}+\cdots,
$$

where the ellipsis represents exponentially suppressed contributions, with a gap that is $O\left(m_{\pi}\right)$. The first exponential corresponds to the on-shell external meson $P$ and represents the state we are interested in. The second exponential corresponds to the state $P+\gamma$ where both the meson and the photon are on-shell and have a total momentum $\boldsymbol{p}$ and a relative momentum $\boldsymbol{k}$. A similar time dependence also appears in the WI from the rotation of the $P$ source; this is the second term on the right-hand side of Eq. (C4). As discussed above, when $\boldsymbol{k} \neq 0$, it is possible to isolate the matrix element corresponding to the state $P$.

The problem we now address is to study the limit $\boldsymbol{k} \rightarrow \mathbf{0}$, paying special attention to the leading cutoff effects. This can be done by using the exact WI satisfied by $C_{A}^{\alpha \mu}(t ; \boldsymbol{k}, \boldsymbol{p})$ at finite lattice spacing; in particular, we aim to understand the structure of the correlation function $C_{A}^{\alpha \mu}(t ; \boldsymbol{k}, \boldsymbol{p})$ at $\boldsymbol{k}=0$. To this end, we consider the two-point correlation functions on the right-hand side of Eq. (C4) when $\alpha$ is a spatial index (the case $\alpha=0$ is similar, but in the following we shall concentrate on the case $\alpha=1,2,3$ ). By setting $E_{\gamma}=0$ in the last term of Eq. (C6), we have

$$
\begin{gathered}
C_{A}^{j}(t ; \boldsymbol{p})=\frac{p^{j} \hat{f}_{P}(\boldsymbol{p}) \hat{G}_{P}(\boldsymbol{p})}{2 \hat{E}_{P}(\boldsymbol{p})} e^{-t \hat{E}_{P}(\boldsymbol{p})}+\ldots, \\
C_{A}^{j}\left(t ; E_{\gamma}=0, \boldsymbol{p}-\boldsymbol{k}\right)=C_{A}^{j}(t, \boldsymbol{p}-\boldsymbol{k})=\frac{(p-k)^{j} \hat{f}_{P}(\boldsymbol{p}-\boldsymbol{k}) \hat{G}_{P}(\boldsymbol{p}-\boldsymbol{k})}{2 \hat{E}_{P}(\boldsymbol{p}-\boldsymbol{k})} e^{-t \hat{E}_{P}(\boldsymbol{p}-\boldsymbol{k})}+\ldots,
\end{gathered}
$$

where the ellipsis represents subleading exponentials (the gap is at least $2 m_{\pi}$ ). In the previous expressions $\hat{f}_{P}(\boldsymbol{p})=f_{P}+O\left(a^{2}\right), \hat{G}_{P}(\boldsymbol{p})=G_{P}+O\left(a^{2}\right)$, and $\hat{E}_{P}(\boldsymbol{p})=E_{P}(\boldsymbol{p})+O\left(a^{2}\right)$ where $f_{P}, G_{P}$, and $E_{P}(\boldsymbol{p})$ are, respectively, the continuum decay constant, the continuum matrix element of the pseudoscalar density used as interpolating operator, $G_{P}=\langle 0|P| P(\boldsymbol{p})\rangle$, and the continuum energy of the meson.

By using the previous two expressions and by differentiating Eq. (C4) with respect to the component $k^{i}$ of $\boldsymbol{k}$ and then setting $\boldsymbol{k}=\mathbf{0}$, we obtain

$$
C_{A}^{j i}(t ; \mathbf{0}, \boldsymbol{p})=\frac{\hat{f}_{P}(\boldsymbol{p}) \hat{G}_{P}(\boldsymbol{p})}{2 \hat{E}_{P}(\boldsymbol{p})} e^{-t \hat{E}_{P}(\boldsymbol{p})}\left\{\delta^{i j}+p^{j}\left[\frac{1}{\hat{f}_{P}(\boldsymbol{p})} \frac{\partial \hat{f}_{P}(\boldsymbol{p})}{\partial p^{i}}+\frac{1}{\hat{G}_{P}(\boldsymbol{p})} \frac{\partial \hat{G}_{P}(\boldsymbol{p})}{\partial p^{i}}-\left(t+\frac{1}{\hat{E}_{P}(\boldsymbol{p})}\right) \frac{\partial \hat{E}_{P}(\boldsymbol{p})}{\partial p^{i}}\right]\right\}+\ldots
$$

where the ellipsis again represents the subleading exponentials and we have used the fact that $-\partial_{k_{i}} g(\boldsymbol{p}-\boldsymbol{k})=$ $\partial_{p_{i}} g(\boldsymbol{p}-\boldsymbol{k})$.

As can be seen, the structure of $C_{A}^{j i}(t ; \mathbf{0}, \boldsymbol{p})$ is highly nontrivial. Note in particular the term linear in $t$ that is a manifestation of the singular behavior at large distances of the correlation function (this generates a double pole in momentum space that is at the original the infrared divergence). An important consequence of the strategy proposed in Sec. IV which we have used in our calculations is that the terms in squared brackets in Eq. (C17) disappear at any value of $\boldsymbol{p}$ when we contract the correlation function with the physical polarization vectors of the photon. With our choice of kinematics, these satisfy the relation

$$
\sum_{\mu=0}^{3} \epsilon_{\mu}^{r}(\boldsymbol{k}) p_{\mu}=\sum_{i=1}^{3} \epsilon_{i}^{r}(\mathbf{0}) p_{i}=0
$$

Indeed, the $H(3)$ symmetry implies that

$$
\begin{aligned}
\frac{\partial \hat{f}_{P}(\boldsymbol{p})}{\partial p^{i}} & =p^{i} \times O\left(a^{2}\right), \quad \frac{\partial \hat{G}_{P}(\boldsymbol{p})}{\partial p^{i}}=p^{i} \times O\left(a^{2}\right), \\
\frac{\partial \hat{E}_{P}(\boldsymbol{p})}{\partial p^{i}} & =\frac{p^{i}}{E_{P}(\boldsymbol{p})}+O\left(a^{2}\right)
\end{aligned}
$$

and thus 


$$
\begin{aligned}
C_{A}^{j r}(t ; \mathbf{0}, \boldsymbol{p}) & =\sum_{i=1}^{3} \epsilon_{i}^{r}(\mathbf{0}) C_{A}^{j i}(t ; \mathbf{0}, \boldsymbol{p}) \\
& =\epsilon_{j}^{r}(\mathbf{0}) \frac{\hat{f}_{P}(\boldsymbol{p}) \hat{G}_{P}(\boldsymbol{p})}{2 \hat{E}_{P}(\boldsymbol{p})} e^{-t \hat{E}_{P}(\boldsymbol{p})}+\ldots
\end{aligned}
$$

We conclude that $C_{A}^{j r}(t ; \mathbf{0}, \boldsymbol{p})$ can be analyzed as expected to extract the coefficient of the leading exponential. We stress that the above demonstration shows that from $C_{A}^{j r}(t ; \mathbf{0}, \boldsymbol{p})$ we can extract precisely the decay constant which appears in the lattice matrix element of the axial current in Eq. (C1), without to have to make a choice for the lattice momentum $p_{L}^{\alpha}$.

\section{APPENDIX D: DETAILS OF THE SIMULATION AND CORRELATION MATRICES FOR THE FORM FACTORS}

In this appendix, we present some numerical information that may be useful to the reader. We start by listing in
Tables I and II the parameters used in our numerical simulations: the values of $\beta$ and the corresponding lattice spacings, the volumes, the quark mass parameters and the corresponding pion masses, $m_{\pi}$, and $m_{\pi} L$, the numbers of configurations, and the twisting angles introduced to inject momenta in the correlation functions.

Given the smooth behavior that we find for the form factors as functions of $x_{\gamma}$ in the region where we have data, for most phenomenological purposes it is sufficient to use form factors obtained using the Ansätze and coefficients given in Sec. V. However, in the tables in Secs. D 1-D 4 below, we also present the values of the form factors, $F_{A}$ and $F_{V}$ at selected values of the photon energy $x_{\gamma}$, for the pion, kaon, $D$ and $D_{s}$ mesons, together with the corresponding uncertainties, $\Delta_{F_{A}}$ and $\Delta_{F_{V}}$. The results have been extrapolated to the continuum and to physical quark masses. We also give the correlation matrices of these results. For the $D$ and $D_{s}$ mesons, for which we only have data in a limited range of $x_{\gamma}$, the results in Secs. D 3 and D 4 were obtained by averaging the results obtained using

TABLE I. There was a mistake in the values of the lattice spacing values of the simulated sea and valence quark bare masses for each ensemble used in this work. The table is the same as in Ref. [15] except for $\mu_{s}$ and $\mu_{c}$ which are

\begin{tabular}{|c|c|c|c|c|c|c|c|}
\hline Ensemble & $\beta$ & $a(\mathrm{fm})$ & $V / a^{4}$ & $a \mu_{\mathrm{sea}}=a \mu_{\ell}$ & $a \mu_{\sigma}$ & $a \mu_{\delta}$ & $N_{c f g}$ \\
\hline A30.32 & 1.90 & $0.0885(36)$ & $32^{3} \times 64$ & 0.0030 & 0.15 & 0.19 & 150 \\
\hline$A 40.32$ & & & & 0.0040 & & & 100 \\
\hline A60.24 & 1.90 & $0.0885(36)$ & $24^{4} \times 48$ & 0.0060 & 0.15 & 0.19 & 150 \\
\hline$A 80.24$ & & & & 0.0080 & & & 150 \\
\hline$B 25.32$ & 1.95 & $0.0815(30)$ & $32^{3} \times 64$ & 0.0025 & 0.135 & 0.170 & 150 \\
\hline B35.32 & & & & 0.0035 & & & 150 \\
\hline B55.32 & & & & 0.0055 & & & 150 \\
\hline$B 75.32$ & & & & 0.0075 & & & 80 \\
\hline$D 15.48$ & 2.10 & $0.0619(18)$ & $48^{3} \times 96$ & 0.0015 & 0.12 & 0.1385 & 100 \\
\hline D20.48 & & & & 0.0020 & & & 100 \\
\hline D30.48 & & & & 0.0030 & & & 100 \\
\hline
\end{tabular}
given in Table II.

TABLE II. Central values of the pion mass $m_{\pi}$, of the lattice size $L$, and of the product $m_{\pi} L$ for the various ensembles used in this work. We also give the values of the angles use to define the $z$-component of the meson and

\begin{tabular}{|c|c|c|c|c|c|c|c|c|}
\hline Ensemble & $\beta$ & $L(\mathrm{fm})$ & $m_{\pi}(\mathrm{MeV})$ & $m_{\pi} L$ & $a \mu_{\mathrm{sea}}=a \mu_{\ell}$ & $a \mu_{s}$ & $a \mu_{c}$ & $\theta_{i=0, s, t}$ \\
\hline A30.32 & 1.90 & 2.84 & 273 & 3.9 & 0.0030 & 0.02363 & 0.27903 & $0,0.2288,0.3432$ \\
\hline$A 40.32$ & & & 315 & 4.5 & 0.0040 & 0.02760 & 0.29900 & $0.6864,0.8580$ \\
\hline$A 60.24$ & 1.90 & 2.13 & 383 & 4.1 & 0.0060 & & & \\
\hline$A 80.24$ & & & 441 & 4.7 & 0.0080 & & & \\
\hline$B 25.32$ & 1.95 & 2.61 & 256 & 3.4 & 0.0025 & 0.02094 & 0.24725 & $0,0.2107,0.3160$ \\
\hline B35.32 & & & 300 & 4.0 & 0.0035 & 0.0239 & 0.267300 & $0.6321,0.7901$ \\
\hline B55.32 & & & 373 & 4.9 & 0.0055 & & & \\
\hline$B 75.32$ & & & 436 & 6.1 & 0.0075 & & & \\
\hline$D 15.48$ & 2.10 & 2.97 & 228 & 3.4 & 0.0015 & 0.01612 & 0.19037, & $0,0.2400,0.3601$, \\
\hline D20.48 & & & 252 & 3.8 & 0.0020 & 0.01910 & 0.20540 & $0.7201,0.9002$ \\
\hline D30.48 & & & 309 & 4.7 & 0.0030 & & & \\
\hline
\end{tabular}
photon momenta, $\boldsymbol{p}=\left(0,0, \frac{2 \pi}{L}\left(\theta_{0}-\theta_{s}\right)\right)$ and $\boldsymbol{k}=\left(0,0, \frac{2 \pi}{L}\left(\theta_{0}-\theta_{t}\right)\right)$, respectively. 
Eqs. (37) and (42) and including the difference between the two Anzätze in the estimate of the uncertainties. As might be expected from Fig. 11 and the accompanying discussion, the extrapolations using the two anzatze diverge significantly at larger $x_{\gamma}$ which is reflected in the growing uncertainties in the results in the tables in Secs. D 3 and D 4.

\section{Results for $\boldsymbol{F}_{\boldsymbol{A}}\left(\boldsymbol{x}_{\gamma}\right)$ and $\boldsymbol{F}_{\boldsymbol{V}}\left(\boldsymbol{x}_{\gamma}\right)$ of the pion}

\begin{tabular}{c|c|c}
$x_{\gamma}$ & $F_{A}$ & $\Delta_{F_{A}}$ \\
\hline 0 & 0.0104088 & 0.00262483 \\
\hline 0.1 & 0.0104435 & 0.00260149 \\
\hline 0.2 & 0.0104782 & 0.0025792 \\
\hline 0.3 & 0.0105129 & 0.00255799 \\
\hline 0.4 & 0.0105477 & 0.00253788 \\
\hline 0.5 & 0.0105824 & 0.00251889 \\
\hline 0.6 & 0.0106171 & 0.00250106 \\
\hline 0.7 & 0.0106519 & 0.00248441 \\
\hline 0.8 & 0.0106866 & 0.00246897 \\
\hline 0.9 & 0.0107213 & 0.00245474 \\
\hline 1 & 0.010756 & 0.00244177
\end{tabular}

\begin{tabular}{c|c|c}
$x_{\gamma}$ & $F_{V}$ & $\Delta_{F_{V}}$ \\
\hline 0 & 0.0233352 & 0.00214581 \\
\hline 0.1 & 0.023309 & 0.0021304 \\
\hline 0.2 & 0.0232828 & 0.00211523 \\
\hline 0.3 & 0.0232566 & 0.00210031 \\
\hline 0.4 & 0.0232303 & 0.00208563 \\
\hline 0.5 & 0.0232041 & 0.00207121 \\
\hline 0.6 & 0.0231779 & 0.00205705 \\
\hline 0.7 & 0.0231517 & 0.00204315 \\
\hline 0.8 & 0.0231254 & 0.00202952 \\
\hline 0.9 & 0.0230992 & 0.00201617 \\
\hline 1 & 0.023073 & 0.0020031
\end{tabular}

$F_{A}$ correlation matrix

$\left.\begin{array}{l|l|l|l|l|l|l|l|l|l|l|}1.000 & 1.000 & 0.999 & 0.998 & 0.997 & 0.995 & 0.992 & 0.989 & 0.986 & 0.982 & 0.977 \\ 1.000 & 1.000 & 1.000 & 0.999 & 0.998 & 0.997 & 0.995 & 0.992 & 0.989 & 0.985 & 0.981 \\ 0.999 & 1.000 & 1.000 & 1.000 & 0.999 & 0.998 & 0.996 & 0.994 & 0.992 & 0.989 & 0.985 \\ 0.998 & 0.999 & 1.000 & 1.000 & 1.000 & 0.999 & 0.998 & 0.996 & 0.994 & 0.992 & 0.988 \\ 0.997 & 0.998 & 0.999 & 1.000 & 1.000 & 1.000 & 0.999 & 0.998 & 0.996 & 0.994 & 0.991 \\ 0.995 & 0.997 & 0.998 & 0.999 & 1.000 & 1.000 & 1.000 & 0.999 & 0.998 & 0.996 & 0.994 \\ 0.992 & 0.995 & 0.996 & 0.998 & 0.999 & 1.000 & 1.000 & 1.000 & 0.999 & 0.998 & 0.996 \\ 0.989 & 0.992 & 0.994 & 0.996 & 0.998 & 0.999 & 1.000 & 1.000 & 1.000 & 0.999 & 0.998 \\ 0.986 & 0.989 & 0.992 & 0.994 & 0.996 & 0.998 & 0.999 & 1.000 & 1.000 & 1.000 & 0.999 \\ 0.982 & 0.985 & 0.989 & 0.992 & 0.994 & 0.996 & 0.998 & 0.999 & 1.000 & 1.000 & 1.000 \\ 0.977 & 0.981 & 0.985 & 0.988 & 0.991 & 0.994 & 0.996 & 0.998 & 0.999 & 1.000 & 1.000\end{array}\right)$

$F_{V}$ correlation matrix

$\left.\begin{array}{l|l|l|l|l|l|l|l|l|l|l}1.000 & 1.000 & 1.000 & 0.999 & 0.999 & 0.999 & 0.998 & 0.997 & 0.996 & 0.995 & 0.994 \\ 1.000 & 1.000 & 1.000 & 1.000 & 0.999 & 0.999 & 0.998 & 0.998 & 0.997 & 0.996 & 0.995 \\ 1.000 & 1.000 & 1.000 & 1.000 & 1.000 & 0.999 & 0.999 & 0.998 & 0.998 & 0.997 & 0.996 \\ 0.999 & 1.000 & 1.000 & 1.000 & 1.000 & 1.000 & 0.999 & 0.999 & 0.998 & 0.998 & 0.997 \\ 0.999 & 0.999 & 1.000 & 1.000 & 1.000 & 1.000 & 1.000 & 0.999 & 0.999 & 0.998 & 0.998 \\ 0.999 & 0.999 & 0.999 & 1.000 & 1.000 & 1.000 & 1.000 & 1.000 & 0.999 & 0.999 & 0.998 \\ 0.998 & 0.998 & 0.999 & 0.999 & 1.000 & 1.000 & 1.000 & 1.000 & 1.000 & 0.999 & 0.999 \\ 0.997 & 0.998 & 0.998 & 0.999 & 0.999 & 1.000 & 1.000 & 1.000 & 1.000 & 1.000 & 0.999 \\ 0.996 & 0.997 & 0.998 & 0.998 & 0.999 & 0.999 & 1.000 & 1.000 & 1.000 & 1.000 & 1.000 \\ 0.995 & 0.996 & 0.997 & 0.998 & 0.998 & 0.999 & 0.999 & 1.000 & 1.000 & 1.000 & 1.000 \\ 0.994 & 0.995 & 0.996 & 0.997 & 0.998 & 0.998 & 0.999 & 0.999 & 1.000 & 1.000 & 1.000\end{array}\right)$


2. Results for $\boldsymbol{F}_{A}\left(x_{\gamma}\right)$ and $\boldsymbol{F}_{V}\left(\boldsymbol{x}_{\gamma}\right)$ of the kaon

\begin{tabular}{c|c|c}
$x_{\gamma}$ & $F_{A}$ & $\Delta_{F_{A}}$ \\
\hline 0 & 0.0370382 & 0.00876335 \\
\hline 0.1 & 0.0369171 & 0.00828189 \\
\hline 0.2 & 0.0367961 & 0.00784116 \\
\hline 0.3 & 0.0366751 & 0.00744839 \\
\hline 0.4 & 0.0365541 & 0.00711155 \\
\hline 0.5 & 0.0364331 & 0.00683889 \\
\hline 0.6 & 0.0363121 & 0.00663833 \\
\hline 0.7 & 0.0361911 & 0.00651654 \\
\hline 0.8 & 0.0360701 & 0.00647795 \\
\hline 0.9 & 0.0359491 & 0.00652404 \\
\hline 1 & 0.035828 & 0.00665305
\end{tabular}

$F_{A}$ correlation matrix

$\left.\begin{array}{l|l|l|l|l|l|l|l|l|l|l}1.000 & 0.998 & 0.990 & 0.975 & 0.951 & 0.916 & 0.869 & 0.808 & 0.736 & 0.654 & 0.566 \\ 0.998 & 1.000 & 0.997 & 0.988 & 0.970 & 0.941 & 0.899 & 0.845 & 0.779 & 0.703 & 0.620 \\ 0.990 & 0.997 & 1.000 & 0.997 & 0.985 & 0.963 & 0.929 & 0.883 & 0.823 & 0.753 & 0.676 \\ 0.975 & 0.988 & 0.997 & 1.000 & 0.996 & 0.982 & 0.957 & 0.918 & 0.867 & 0.805 & 0.734 \\ 0.951 & 0.970 & 0.985 & 0.996 & 1.000 & 0.995 & 0.979 & 0.950 & 0.909 & 0.855 & 0.793 \\ 0.916 & 0.941 & 0.963 & 0.982 & 0.995 & 1.000 & 0.994 & 0.976 & 0.946 & 0.902 & 0.849 \\ 0.869 & 0.899 & 0.929 & 0.957 & 0.979 & 0.994 & 1.000 & 0.994 & 0.975 & 0.943 & 0.900 \\ 0.808 & 0.845 & 0.883 & 0.918 & 0.950 & 0.976 & 0.994 & 1.000 & 0.993 & 0.974 & 0.943 \\ 0.736 & 0.779 & 0.823 & 0.867 & 0.909 & 0.946 & 0.975 & 0.993 & 1.000 & 0.994 & 0.975 \\ 0.654 & 0.703 & 0.753 & 0.805 & 0.855 & 0.902 & 0.943 & 0.974 & 0.994 & 1.000 & 0.994 \\ 0.566 & 0.620 & 0.676 & 0.734 & 0.793 & 0.849 & 0.900 & 0.943 & 0.975 & 0.994 & 1.000\end{array}\right)$

\begin{tabular}{c|c|c}
$x_{\gamma}$ & $F_{V}$ & $\Delta_{F_{V}}$ \\
\hline 0 & 0.12439 & 0.00960998 \\
\hline 0.1 & 0.121998 & 0.00891388 \\
\hline 0.2 & 0.119606 & 0.00828371 \\
\hline 0.3 & 0.117214 & 0.0077356 \\
\hline 0.4 & 0.114821 & 0.0072881 \\
\hline 0.5 & 0.112429 & 0.00696062 \\
\hline 0.6 & 0.110037 & 0.00677062 \\
\hline 0.7 & 0.107645 & 0.00672974 \\
\hline 0.8 & 0.105253 & 0.00684066 \\
\hline 0.9 & 0.102861 & 0.00709626 \\
\hline 1 & 0.100469 & 0.00748173
\end{tabular}

$\left.\begin{array}{l|l|l|l|l|l|l|l|l|l|l|l}1.000 & 0.997 & 0.985 & 0.961 & 0.921 & 0.860 & 0.777 & 0.674 & 0.558 & 0.435 & 0.316 \\ 0.997 & 1.000 & 0.996 & 0.980 & 0.949 & 0.898 & 0.825 & 0.731 & 0.622 & 0.506 & 0.391 \\ 0.985 & 0.996 & 1.000 & 0.994 & 0.974 & 0.935 & 0.874 & 0.791 & 0.692 & 0.583 & 0.474 \\ 0.961 & 0.980 & 0.994 & 1.000 & 0.993 & 0.967 & 0.921 & 0.852 & 0.765 & 0.666 & 0.565 \\ 0.921 & 0.949 & 0.974 & 0.993 & 1.000 & 0.991 & 0.961 & 0.909 & 0.837 & 0.752 & 0.661 \\ 0.860 & 0.898 & 0.935 & 0.967 & 0.991 & 1.000 & 0.989 & 0.957 & 0.903 & 0.834 & 0.756 \\ 0.777 & 0.825 & 0.874 & 0.921 & 0.961 & 0.989 & 1.000 & 0.989 & 0.956 & 0.905 & 0.843 \\ 0.674 & 0.731 & 0.791 & 0.852 & 0.909 & 0.957 & 0.989 & 1.000 & 0.989 & 0.958 & 0.914 \\ 0.558 & 0.622 & 0.692 & 0.765 & 0.837 & 0.903 & 0.956 & 0.989 & 1.000 & 0.990 & 0.964 \\ 0.435 & 0.506 & 0.583 & 0.666 & 0.752 & 0.834 & 0.905 & 0.958 & 0.990 & 1.000 & 0.992 \\ 0.316 & 0.391 & 0.474 & 0.565 & 0.661 & 0.756 & 0.843 & 0.914 & 0.964 & 0.992 & 1.000\end{array}\right)$




\section{Results for $\boldsymbol{F}_{A}\left(\boldsymbol{x}_{\gamma}\right)$ and $\boldsymbol{F}_{V}\left(\boldsymbol{x}_{\gamma}\right)$ of the $\boldsymbol{D}$ meson}

\begin{tabular}{|c|c|c|c|c|c|c|c|c|c|c|c|c|c|}
\hline$x_{\gamma}$ & 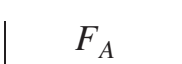 & & \multicolumn{11}{|c|}{$F_{A}$ correlation matrix } \\
\hline 0 & 0.11029 & 00885428 & 1.000 & 0.907 & 0.704 & 0.491 & 0.314 & 0.187 & 0.102 & 0.049 & 0.015 & -0.006 & -0.020 \\
\hline 0.1 & 0988347 & 0.00740568 & 0.907 & 1.000 & 0.931 & 0.776 & 0.598 & 0.439 & 0.314 & 0.223 & 0156 & 0.108 & 0.072 \\
\hline .2 & 0887281 & 00727782 & 0 & 0.931 & .000 & 948 & 0 & 0 & 4 & 465 & 387 & 0.327 & 0.281 \\
\hline .3 & 0.079585 & 868 & 0.491 & 0.776 & 0.948 & 1.000 & 0.961 & 0.875 & 0.781 & 0.697 & 0.627 & 0.570 & 0.524 \\
\hline .4 & 0.0711547 & 365 & 0.314 & 508 & 827 & 0.961 & 000 & 0074 & 1 & 863 & 0 & 0.764 & 726 \\
\hline .5 & 0.063267 & 973 & 0.187 & 0.439 & 0.688 & 0.875 & 0.974 & 1.000 & 0.985 & 0.954 & 0.920 & 0000 & 0.859 \\
\hline 6 & 558021 & 0.0137148 & 0.102 & 0.314 & 0.564 & 0.781 & 0.921 & 0.985 & 1.000 & 0.991 & 0.974 & 0.953 & 0.934 \\
\hline 0.7 & 0.0486731 & & 0.049 & 0.223 & .465 & 0.697 & 0.863 & 0.954 & 0.991 & 1.000 & 00 & 0.985 & 0.972 \\
\hline .8 & 4 & U. & 0.015 & 0.156 & .387 & 0.627 & 0.810 & 0.920 & 0. & 0.995 & 0 & 0 . & 0.991 \\
\hline 0.9 & 0.0351801 & 2 & -0.006 & 0.108 & 0.327 & 0.570 & 764 & 0.888 & 0.953 & 0.985 & 0.997 & 1.000 & 0.998 \\
\hline 1 & 0.0287293 & 0.027459 & -0.020 & 0.072 & 0.281 & 0.524 & 0.726 & 0.859 & 0.934 & 0.972 & 0.991 & 0.998 & 1.000 \\
\hline
\end{tabular}

\begin{tabular}{|c|c|c|c|c|c|c|c|c|c|c|c|c|c|}
\hline & $F_{V}$ & & & & & & $F_{V} \mathrm{C}$ & & & & & & \\
\hline 0 & -0.150 & 2. & 1.000 & 0.925 & 0.749 & 0.552 & 0.377 & 0.239 & 0.140 & 0.072 & 0.027 & -0.003 & -0.02 \\
\hline 1 & 0.1 & & 0.925 & 1.000 & 0.941 & 0.807 & 0.647 & 0.494 & 0.364 & 0.263 & 0.187 & 0.130 & 0.087 \\
\hline & -0.12 & & 749 & 0.941 & 1.000 & 0.956 & 085 & 0.721 & .596 & 0.488 & 401 & 0331 & 276 \\
\hline & -0.1 & & 0.552 & 0.807 & 0.956 & 1.000 & 0.966 & 885 & 789 & 0.696 & .015 & 0.541 & 192 \\
\hline & 0 & & 0.377 & 0.647 & 0.851 & 0.966 & .000 & 0.975 & 0.918 & 0.852 & 0.788 & 0.732 & 0.684 \\
\hline .5 & .0 & & 0.239 & 0 & 0 & ( & 5 & 0 & 3 & 0 & 0.904 & 862 & 4 \\
\hline 6 & 13 & & 10 & 4 & 0.596 & 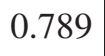 & 8 & 3 & 1.0 & 0.989 & 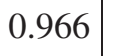 & 0.939 & 0.913 \\
\hline 7 & 6 & 6 & 0.072 & 0.263 & 0.488 & 0.696 & 0.852 & 0.946 & 0.989 & 1.000 & 0.993 & 0.979 & 0.962 \\
\hline 98 & 0.0 & 0.0251904 & 0.027 & 0.187 & 0.401 & 0.615 & 0.788 & 0.904 & 0.966 & 0.99 & 1.000 & 0.996 & 0.987 \\
\hline 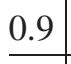 & 0.0 & & -0.003 & 0.130 & 0.331 & 0.547 & 0.732 & 0.862 & 0.939 & 0.979 & 0.996 & 1.000 & 0.997 \\
\hline 1 & -0.0466964 & 0339692 & -0.023 & 0.087 & 0.276 & 0.492 & 0.684 & 0.824 & 0.913 & 0.962 & 0.987 & 0.997 & 1.000 \\
\hline
\end{tabular}


4. Results for $F_{A}\left(x_{\gamma}\right)$ and $F_{V}\left(x_{\gamma}\right)$ of the $D_{s}$ meson

$\left.\begin{array}{c|c|c|c|c|c|c|c|c|c|c|c|c|c}x_{\gamma} & F_{A} & \Delta_{F_{A}} \\ \hline 0 & 0.09307 & 0.00598514 \\ \hline 0.1 & 0.0849608 & 0.00486963 & 0.959 & 1.000 & 0.966 & 0.861 & 0.664 & 0.423 & 0.224 & 0.091 & 0.006 & -0.048 & -0.084 \\ \hline 0.2 & 0.0776688 & 0.00422644 & 0.862 & 0.966 & 1.000 & 0.957 & 0.802 & 0.569 & 0.357 & 0.206 & 0.105 & 0.037 & -0.010 \\ \hline 0.3 & 0.0709899 & 0.00389515 & 0.725 & 0.861 & 0.957 & 1.000 & 0.937 & 0.768 & 0.583 & 0.437 & 0.334 & 0.261 & 0.208 \\ \hline 0.4 & 0.0647833 & 0.00400167 & 0.537 & 0.664 & 0.802 & 0.937 & 1.000 & 0.942 & 0.824 & 0.713 & 0.626 & 0.561 & 0.512 \\ \hline 0.5 & 0.0589483 & 0.00469896 & 0.338 & 0.423 & 0.569 & 0.768 & 0.942 & 1.000 & 0.966 & 0.906 & 0.848 & 0.802 & 0.764 \\ \hline 0.6 & 0.0534115 & 0.00595597 & 0.186 & 0.224 & 0.357 & 0.583 & 0.824 & 0.966 & 1.000 & 0.984 & 0.956 & 0.928 & 0.903 \\ \hline 0.7 & 0.0481175 & 0.00763605 & 0.091 & 0.091 & 0.206 & 0.437 & 0.713 & 0.906 & 0.984 & 1.000 & 0.993 & 0.979 & 0.965 \\ \hline 0.8 & 0.0430239 & 0.00962076 & 0.034 & 0.006 & 0.105 & 0.334 & 0.626 & 0.848 & 0.956 & 0.993 & 1.000 & 0.996 & 0.989 \\ \hline 0.9 & 0.0380979 & 0.0118319 \\ \hline 1 & 0.0333133 & 0.0142191 & 0.001 & -0.048 & 0.037 & 0.261 & 0.561 & 0.802 & 0.928 & 0.979 & 0.996 & 1.000 & 0.998 \\ -0.020 & -0.084 & -0.010 & 0.208 & 0.512 & 0.764 & 0.903 & 0.965 & 0.989 & 0.998 & 1.000\end{array}\right)$

$\left.\begin{array}{c|c|c|c|c|c|c|c|c|c|c|c|c|c}x_{\gamma} & F_{V} & \Delta_{F_{V}} \\ \hline 0 & -0.120018 & 0.0155225 & (1.000 & 0.933 & 0.886 & 0.898 & 0.782 & 0.543 & 0.379 & 0.288 & 0.236 & 0.205 & 0.184 \\ \hline 0.1 & -0.0989568 & 0.0117214 & 0.933 & 1.000 & 0.989 & 0.935 & 0.667 & 0.317 & 0.107 & -0.004 & -0.066 & -0.103 & -0.127 \\ \hline 0.2 & -0.0824261 & 0.00951697 & 0.886 & 0.989 & 1.000 & 0.955 & 0.679 & 0.314 & 0.093 & -0.024 & -0.090 & -0.130 & -0.157 \\ \hline 0.3 & -0.0684115 & 0.00783577 & 0.898 & 0.935 & 0.955 & 1.000 & 0.862 & 0.571 & 0.369 & 0.255 & 0.188 & 0.147 & 0.119 \\ \hline 0.4 & -0.05594 & 0.00806476 & 0.782 & 0.667 & 0.679 & 0.862 & 1.000 & 0.908 & 0.787 & 0.707 & 0.656 & 0.623 & 0.600 \\ \hline 0.5 & -0.0444834 & 0.0110619 & 0.543 & 0.317 & 0.314 & 0.571 & 0.908 & 1.000 & 0.973 & 0.938 & 0.912 & 0.893 & 0.879 \\ \hline 0.6 & -0.03373 & 0.0158477 & 0.379 & 0.107 & 0.093 & 0.369 & 0.787 & 0.973 & 1.000 & 0.993 & 0.982 & 0.972 & 0.965 \\ \hline 0.7 & -0.0234841 & 0.0215682 & 0.288 & -0.004 & -0.024 & 0.255 & 0.707 & 0.938 & 0.993 & 1.000 & 0.998 & 0.993 & 0.990 \\ \hline 0.8 & -0.0136163 & 0.0278383 \\ \hline 0.9 & -0.00403801 & 0.0344741 \\ \hline 1 & 0.00531392 & 0.0413737 & 0.236 & -0.066 & -0.090 & 0.188 & 0.656 & 0.912 & 0.982 & 0.998 & 1.000 & 0.999 & 0.997 \\ 0.184 & -0.127 & -0.157 & 0.119 & 0.600 & 0.879 & 0.965 & 0.990 & 0.997 & 1.000 & 1.000\end{array}\right)$

\section{APPENDIX E: CORRELATION MATRICES OF THE COEFFICIENTS $C_{A, V}^{P}$ AND $D_{A, V}^{P}$}

(i) Pion

$$
\begin{aligned}
& C_{A}^{\pi}=0.0104 \pm 0.0026 \\
& C_{V}^{\pi}=0.0233 \pm 0.0021 \\
& D_{A}^{\pi}=0.00035 \pm 0.00057 \\
& D_{V}^{\pi}=-0.00026 \pm 0.00027
\end{aligned}
$$

\begin{tabular}{c|c|c|c|c} 
& $C_{A}^{\pi}$ & $C_{V}^{\pi}$ & $D_{A}^{\pi}$ & $D_{V}^{\pi}$ \\
\hline$C_{A}^{\pi}$ & 1.000 & 0.323 & -0.419 & -0.185 \\
\hline$C_{V}^{\pi}$ & 0.323 & 1.000 & -0.444 & -0.570 \\
\hline$D_{A}^{\pi}$ & -0.419 & -0.444 & 1.000 & 0.523 \\
\hline$D_{V}^{\pi}$ & -0.185 & -0.570 & 0.523 & 1.000
\end{tabular}


(ii) Kaon

$$
\begin{aligned}
& C_{A}^{K}=0.0370 \pm 0.0088 \\
& C_{V}^{K}=0.1244 \pm 0.0096 \\
& D_{A}^{K}=-0.0012 \pm 0.0074 \\
& D_{V}^{K}=-0.02 \pm 0.01
\end{aligned}
$$

\begin{tabular}{c|cccc} 
& $C_{A}^{K}$ & $C_{V}^{K}$ & $D_{A}^{K}$ & $D_{V}^{K}$ \\
\hline$C_{A}^{K}$ & 1.000 & 0.027 & -0.673 & 0.067 \\
\hline$C_{V}^{K}$ & 0.027 & 1.000 & 0.032 & -0.714 \\
\hline$D_{A}^{K}$ & -0.673 & 0.032 & 1.000 & -0.193 \\
\hline$D_{V}^{K}$ & 0.067 & -0.714 & -0.193 & 1.000
\end{tabular}

(iii) D-meson (linear)

$$
\begin{aligned}
& C_{A}^{D}=0.1090 \pm 0.0087 \\
& C_{V}^{D}=-0.148 \pm 0.014 \\
& D_{A}^{D}=-0.10 \pm 0.03 \\
& D_{V}^{D}=0.123 \pm 0.041
\end{aligned}
$$

\begin{tabular}{c|cccc} 
& $C_{A}^{D}$ & $C_{V}^{D}$ & $D_{A}^{D}$ & $D_{V}^{D}$ \\
\hline$C_{A}^{D}$ & 1.000 & -0.234 & -0.557 & 0.217 \\
\hline$C_{V}^{D}$ & -0.234 & 1.000 & 0.143 & -0.580 \\
\hline$D_{A}^{D}$ & -0.557 & 0.143 & 1.000 & -0.209 \\
\hline$D_{V}^{D}$ & 0.217 & -0.580 & -0.209 & 1.000
\end{tabular}

(iv) D-meson (pole)

$$
\begin{aligned}
& \tilde{C}_{A}^{D}=0.1115 \pm 0.0088 \\
& \tilde{C}_{V}^{D}=-0.153 \pm 0.015 \\
& \tilde{D}_{A}^{D}=1.3 \pm 0.4 \\
& \tilde{D}_{V}^{D}=1.23 \pm 0.44
\end{aligned}
$$

\begin{tabular}{c|cccc} 
& $\tilde{C}_{A}^{D}$ & $\tilde{C}_{V}^{D}$ & $\tilde{D}_{A}^{D}$ & $\tilde{D}_{V}^{D}$ \\
\hline$\tilde{C}_{A}^{D}$ & 1.000 & -0.259 & 0.346 & 0.199 \\
\hline$\tilde{C}_{V}^{D}$ & -0.259 & 1.000 & -0.105 & -0.383 \\
\hline$\tilde{D}_{A}^{D}$ & 0.346 & -0.105 & 1.000 & 0.173 \\
\hline$\tilde{D}_{V}^{D}$ & 0.199 & -0.383 & 0.173 & 1.000
\end{tabular}

(v) $\mathrm{D}_{s}$-meson (linear)

$$
\begin{aligned}
& C_{A}^{D_{s}}=0.0923 \pm 0.0058 \\
& C_{V}^{D_{s}}=-0.116 \pm 0.014 \\
& D_{A}^{D_{s}}=-0.071 \pm 0.013 \\
& D_{V}^{D_{s}}=0.160 \pm 0.029
\end{aligned}
$$

\begin{tabular}{l|cccc} 
& $C_{A}^{D_{s}}$ & $C_{V}^{D_{s}}$ & $D_{A}^{D_{s}}$ & $D_{V}^{D_{s}}$ \\
\hline$C_{A}^{D_{s}}$ & 1.000 & -0.454 & -0.745 & 0.544 \\
\hline$C_{V}^{D_{s}}$ & -0.454 & 1.000 & 0.474 & -0.900 \\
\hline$D_{A}^{D_{s}}$ & -0.745 & 0.474 & 1.000 & -0.721 \\
\hline$D_{V}^{D_{s}}$ & 0.544 & -0.900 & -0.721 & 1.000
\end{tabular}

(vi) $\mathrm{D}_{s}$-meson (pole)

$$
\begin{aligned}
& \tilde{C}_{A}^{D_{s}}=0.094 \pm 0.006 \\
& \tilde{C}_{V}^{D_{s}}=-0.124 \pm 0.015 \\
& \tilde{D}_{A}^{D_{s}}=1.07 \pm 0.19 \\
& \tilde{D}_{V}^{D_{s}}=2.64 \pm 0.24
\end{aligned}
$$

\begin{tabular}{l|cccc} 
& $\tilde{C}_{A}^{D_{s}}$ & $\tilde{C}_{V}^{D_{s}}$ & $\tilde{D}_{A}^{D_{s}}$ & $\tilde{D}_{V}^{D_{s}}$ \\
\hline$\tilde{C}_{A}^{D_{s}}$ & 1.000 & -0.444 & 0.546 & 0.477 \\
\hline$\tilde{C}_{V}^{D_{s}}$ & -0.444 & 1.000 & -0.335 & -0.373 \\
\hline$\tilde{D}_{A}^{D_{s}}$ & 0.546 & -0.335 & 1.000 & 0.681 \\
\hline$\tilde{D}_{V}^{D_{s}}$ & 0.477 & -0.373 & 0.681 & 1.000
\end{tabular}


[1] M. Tanabashi et al. (Particle Data Group), Phys. Rev. D 98, 030001 (2018).

[2] F. Bloch and A. Nordsieck, Phys. Rev. 52, 54 (1937).

[3] S. Weinberg, The Quantum Theory of Fields. Vol. 1: Foundations (Cambridge University Press, Cambridge, England, 1995).

[4] F. E. Low, Phys. Rev. 96, 1428 (1954).

[5] J. Bijnens and P. Talavera, Nucl. Phys. B489, 387 (1997).

[6] C. Q. Geng, I. L. Ho, and T. H. Wu, Nucl. Phys. B684, 281 (2004).

[7] V. Mateu and J. Portoles, Eur. Phys. J. C 52, 325 (2007).

[8] R. Unterdorfer and H. Pichl, Eur. Phys. J. C 55, 273 (2008).

[9] V. Cirigliano, G. Ecker, H. Neufeld, A. Pich, and J. Portoles, Rev. Mod. Phys. 84, 399 (2012).

[10] N. Carrasco, V. Lubicz, G. Martinelli, C. T. Sachrajda, N. Tantalo, C. Tarantino, and M. Testa, Phys. Rev. D 91, 074506 (2015).

[11] V. Lubicz, G. Martinelli, C. T. Sachrajda, F. Sanfilippo, S. Simula, and N. Tantalo, Phys. Rev. D 95, 034504 (2017).

[12] V. Lubicz, G. Martinelli, C. T. Sachrajda, F. Sanfilippo, S. Simula, N. Tantalo, and C. Tarantino, Proc. Sci., LATTICE2016 (2016) 290 [arXiv:1610.09668].

[13] N. Tantalo, V. Lubicz, G. Martinelli, C. T. Sachrajda, F. Sanfilippo, and S. Simula, arXiv:1612.00199.

[14] D. Giusti, V. Lubicz, G. Martinelli, C. T. Sachrajda, F. Sanfilippo, S. Simula, N. Tantalo, and C. Tarantino, Phys. Rev. Lett. 120, 072001 (2018).

[15] M. Di Carlo, D. Giusti, V. Lubicz, G. Martinelli, C. T. Sachrajda, F. Sanfilippo, S. Simula, and N. Tantalo, Phys. Rev. D 100, 034514 (2019).

[16] G. M. de Divitiis et al., J. High Energy Phys. 04 (2012) 124.

[17] G. M. de Divitiis, R. Frezzotti, V. Lubicz, G. Martinelli, R. Petronzio, G. C. Rossi, F. Sanfilippo, S. Simula, and N. Tantalo (RM123 Collaboration), Phys. Rev. D 87, 114505 (2013).

[18] D. Becirevic, B. Haas, and E. Kou, Phys. Lett. B 681, 257 (2009).

[19] N. Carrasco et al. (European Twisted Mass Collaboration), Nucl. Phys. B887, 19 (2014).
[20] G. M. de Divitiis et al., arXiv:1908.10160.

[21] C. Kane, C. Lehner, S. Meinel, and A. Soni, Proc. Sci., LATTICE2019 (2019) 134 [arXiv:1907.00279].

[22] J. Bijnens, G. Ecker, and J. Gasser, Nucl. Phys. B396, 81 (1993).

[23] M. Beneke, V. M. Braun, Y. Ji, and Y. B. Wei, J. High Energy Phys. 07 (2018) 154.

[24] L. Maiani and M. Testa, Phys. Lett. B 245, 585 (1990).

[25] G. M. de Divitiis, R. Petronzio, and N. Tantalo, Phys. Lett. B 595, 408 (2004).

[26] J. M. Flynn, A. Juttner, C. T. Sachrajda, P. A. Boyle, and J. M. Zanotti, J. High Energy Phys. 05 (2007) 016.

[27] R. Sommer, Nucl. Phys. B411, 839 (1994).

[28] J. Bijnens and G. Ecker, Annu. Rev. Nucl. Part. Sci. 64, 149 (2014).

[29] L. Del Debbio, L. Giusti, M. Lüscher, R. Petronzio, and N. Tantalo, J. High Energy Phys. 02 (2007) 082.

[30] X. Feng, S. Aoki, H. Fukaya, S. Hashimoto, T. Kaneko, J. i. Noaki, and E. Shintani, Phys. Rev. Lett. 109, 182001 (2012).

[31] N. Carrasco et al., Phys. Rev. D 91, 054507 (2015).

[32] B. Blossier et al. (ETM Collaboration), J. High Energy Phys. 04 (2010) 049.

[33] V. Barger, C. W. Chiang, W. Y. Keung, and D. Marfatia, Phys. Rev. Lett. 108, 081802 (2012).

[34] B. Batell, D. McKeen, and M. Pospelov, Phys. Rev. Lett. 107, 011803 (2011).

[35] J. Albrecht, E. Stamou, R. Ziegler, and R. Zwicky, arXiv: 1911.05018.

[36] M. Hayakawa and S. Uno, Prog. Theor. Phys. 120, 413 (2008).

[37] R. Frezzotti, P. A. Grassi, S. Sint, and P. Weisz (Alpha Collaboration), J. High Energy Phys. 08 (2001) 058.

[38] R. Frezzotti and G. C. Rossi, J. High Energy Phys. 08 (2004) 007.

[39] R. Frezzotti and G. C. Rossi, J. High Energy Phys. 10 (2004) 070.

[40] M. Bochicchio, L. Maiani, G. Martinelli, G. C. Rossi, and M. Testa, Nucl. Phys. B262, 331 (1985). 